\title{
The Nature of Space and of Gravitation
}

\author{
Jacob Schaff \\ Instituto de Fsica, Universidade Federal do Rio Grande do Sul (UFRGS), Porto Alegre, Brazil \\ Email: schaf@if.ufrgs.br
}

Received May 12, 2012; revised June 8, 2012; accepted July 1, 2012

\begin{abstract}
Many recent highly precise and unmistakable observational facts achieved thanks to the tightly synchronized clocks of the GPS, provide consistent evidence that the gravitational fields are created by velocity fields of real space itself, a vigorous and very stable quantum fluid like spatial medium, the same space that rules the propagation of light and the inertial motion of matter. It is shown that motion of this real space in the ordinary three dimensions round the Earth, round the Sun and round the galactic centers throughout the universe, according to velocity fields closely consistent with the local main astronomical motions, correctly induces the gravitational dynamics observed within these gravitational fields. In this spacedynamics the astronomical bodies all closely rest with respect to the real space, which forthrightly leads to the observed null results of the Michelson light anisotropy experiments as well as to the absence of effects of the solar and galactic gravitational fields on the rate of clocks moving with Earth as recently discovered with the help of the GPS clocks. This spacedynamics exempts us from explaining the circular orbital motions of the planets round the Sun, likewise the rotation of Earth exempted people from explaining the diurnal transit of the heavens in the days of Copernicus and Galileo, because it is space itself that so moves. This spacedynamics also eliminates the need of dark matter and dark energy to explain respectively the galactic gravitational dynamics and the accelerated expansion of the universe. It also straightforwardly accounts in terms of well known and genuine physical effects for all the other observed effects, caused by the gravitational fields on the velocity of light and on the rate of clocks, including all the new effects recently discovered with the help of the GPS. It moreover simulates the non-Euclidean metric underlying Einstein's spacetime curvature. This spacedynamics is the crucial innovation in the current world conception that definitively resolves all at once the troubles afflicting the current theories of space and gravitation.
\end{abstract}

Keywords: Gravitation; Gravitational Physics; Gravitational Effects; Dark Matter; Dark Energy

\section{Introduction}

In Newton's universal gravitation the motion of the planets round the Sun and the free-fall of bodies on Earth is explained in terms of attractive gravitational forces. However, as there is clearly no way to transmit such enormous forces between the planets and the Sun, Newton only wrote that everything happens as if such forces were acting. Although these gravitational forces can explain many observational facts, this explanation is clearly fictitious because the gravitational forces cannot be real.

If the Newtonian gravitational forces do not exist, then the planetary motions as well as the free-fall of bodies released within the earthbased laboratories correspond necessarily to their inertial motion. This incontestably proves that earthbased laboratories are not inertial references. This non-inertial character can only arise from kinematical circumstances in which these laboratories are moving under an upward acceleration of $9.8 \mathrm{~m} / \mathrm{sec}^{2}$ due to upward forces exerted by the earthsurface. As however the geometrical distance of these laboratories from the earth center does not increase with time, this upward acceleration can only be of the centripetal nature because the only accelerations that can act continuously under zero velocity along their instantaneous direction are centripetal accelerations. Somehow each apparently resting earthbased laboratory is moving within the local inertial reference (IR) along a circular path about an overhead axis. As however the earthbased laboratories go on resting, this can make a sense only if the local inertial reference is itself rotating oppositely at the same rate about the same overhead axis so that the earthbased laboratories become apparently immovable however under an upward centripetal force. The kinematical implications of this inertial reference dynamics have drastic consequences in the physical meaning of the astronomical motions. Please see Section 4 for the genuine and explicit practical realization of this dynamics.

In his interpretation of the null results of the Michelson light anisotropy experiments Einstein [1,2] has completely overlooked the kinematical implications of this inertial reference dynamics and its repercussions on the astronomical motions within the solar system and within 
the galaxy as well as on the kinematical state of the earthbased laboratories wherein these experiments have been carried out. It seems obvious that before drawing conclusions from the observed null light anisotropy, it is necessary to settle first the gravitational physics in the solar system, in the galaxy and the origin of the noninertial character of the earthbased laboratories. This is a crucial pre-requisite to a minimally reliable interpretation not only of the Michelson light anisotropy experiments however also of all experiments involving motions of electromagnetic signals and or particles within gravitational fields.

In Einstein's general relativity (GR) [1,2] the resting earthbased laboratories too are under an upward acceleration. However, in GR the nature of this upward acceleration is not centripetal. GR simply assumes that within the gravitational fields all the local inertial references are inherently free falling without appointing any physical reason for it. Instead of looking for the physical cause of this inherent free-fall, Einstein modeled it into the geometry of spacetime. As time inexorably flows, bodies advance along the geodesic lines in the curved spacetime geometry, which in the case of a free body within the earthbased laboratories corresponds to the observed freefall. The geodesic motion pretends to be a generalization of the principle of inertia and of Fermat's principle. Einstein's GR geometrizes gravity and thereby provides only a cleverly tuned spacetime curvature that in fact is nothing more than a mere mathematical account. Despite being able to predict correctly the orbital motions of bodies within the gravitational fields, it does not bring to light the genuine physical origin of gravity. Moreover, now an increasing number of new and unmistakable experimental facts, achieved thanks to the tight synchronization of the clocks of the Global Positioning System (GPS), cannot be explained by GR. Please see the forthcoming Section 2 for details. These facts show that the explanation of gravity in terms of spacetime geometry is insufficient. These findings provide strong evidence that the explanation of gravity in terms of spacetime curvature is illusive and fictitious as much as the explanation in terms of the Newtonian gravitational forces.

According to the Standard Elementary Particle Model, gravity is the weakest among the four forces of nature. Currently, the understanding of the gravitational physics is being attempted from two completely distinct viewpoints that are incompatible with each other. While in Quantum Gravity (QG) the gravitational interaction is imputed to real forces created by the quantum exchange interaction mediated by gravitons, in Einstein's GR the gravitational pull is seen as an inertial effect within curved spacetime. Obviously, if the downward gravitational pull on bodies, observed within the earthbased laboratories, is an inertial effect, these earthbased laboratories must be accelerated upward. However, people are now struggling since four decades for a solution of the dichotomy between the views of GR and or QG without success. While the predictions of QG have not been confirmed by experiments, many new observational facts, achieved with the help of the GPS, are putting in check fundamental postulates of the theory of relativity. Visibly the flaws that have disqualified Newton's gravitational theory and now are raising the endless troubles afflicting the current gravitational theories are the fruit of employing wrong concepts of space. To acknowledge the right nature of space is a crucial issue because it definitely decides the interpretation that can be given to our observations in general and in particular to the effects of the gravitational fields. In Newton's universal gravitation space is static, Euclidean and absolute. Therefore he was forced to invent the gravitational forces to explain gravitation. In Einstein's GR space becomes flexible. It however becomes static as soon as the astronomical bodies stop moving and hence still goes on inactive and static. Therefore he had to invent the spacetime curvature to explain the orbital motions of the planets and the free-fall of bodies. With a wrong notion of space, only fake and fictitious explanations are possible and even to explain the impossible may become necessary. In view of these hopeless perspectives to solve the imbroglio that has become gravitation within the current views, it is vital and urgent to look for a viable alternative.

The strategy that will be followed in the present work is to clear up first the gravitational physics and only then to look for the reason of the null results of the Michelson light anisotropy experiments as well as for the origin of all the other observed effects caused by the gravitational fields on the velocity of light and on the rate of clocks. To this purpose a completely new conception will be developed in which real space is effectively dynamic and moving in the ordinary three dimensions according to a velocity field consistently with the local main astronomical motions. This real space, the same space that rules the propagation of light and the inertial motion of bodies in our laboratories is moving through these laboratories and round the Earth, round the Sun and round all the matter concentrations throughout the universe according to a Keplerian velocity field consistent with the local main astronomical motions. This real space is a powerful and active spatial medium that is the physical support of all the material phenomena and hence is the ultimate but moving reference for rest and for motions of matter and light. Hence motion of the real space is a typical gauge invariant. Only variations of its velocity affect the motion of matter and light. The fact that the velocity of space in this Keplerian velocity field increases for decreasing distance from the gravitational 
center is the key property that engenders the peculiar inertial dynamics (gravitational dynamics) observed within the gravitational fields. Motion of bodies with respect to the local moving real space is not absolute motion in the pre-relativistic sense, because the real space, the ultimate reference for rest and for motion is itself moving. Although a unique and universal absolute reference for rest and for motion is excluded, motion of space within the velocity fields and motions of matter with respect to this moving real space still have a well defined meaning. However, the velocity of a body with respect to the real space is only locally well defined. Moreover, velocity of a body with respect to another body is equal to the usual relative velocity only if the two bodies are very close to each other.

Motion of the real space consistently with the astronomical motions is a clear exigency of the null results of the Michelson light anisotropy experiments and also is perfectly corroborated by many new experimental observations achieved with the help of the GPS as will be described in Section 2. This velocity field plays a fundamental role in the life of the universe and by the first time allows for a straightforward and realistic implementation of Einstein's local equivalence of gravitational and inertial effects. In this spacedynamics the gravitational pull on us is a genuine inertial effect. Moreover, according to this spacedynamics the planets in the solar system, the stars in the galaxy etc. are all closely commoving with the real space and hence are nearly resting with respect to it. Hence, this spacedynamics directly predicts the null results of the Michelson light anisotropy experiments searching specifically for light anisotropy due to the orbital and cosmic motion of Earth as well as the absence of effects of the solar gravitational field on the clocks moving with Earth. It also predicts the small constant light anisotropy of nearly $8 \mathrm{~km} / \mathrm{sec}$ along a fixed horizontal direction with respect to each earthbased laboratory round the earth globe as found by the most sensitive Michelson experiments and corroborated recently by measurements of the one way velocity of light, achieved with the help of the tightly synchronized clocks of the GPS [3]. This spacedynamics automatically explains the circular orbital motions of the planets round the Sun and exempts us from explaining these motions analogously as the rotation of Earth exempted people from explaining the diurnal transit of the heavens in the days of Copernicus and Galileo, because it is space itself that so moves. From this viewpoint the planets move round the Sun along nearly circular equatorial orbits, not because of constraints put by gravitational forces but are simply carried around by the moving space itself and hence are not and need not to move by themselves. The observed orbital motion represents the state of rest with respect to the real space. Visibly the astronomical mo- tions throughout the universe are dominantly motions of space itself. Only the much smaller effects that cause the ellipticity of the orbits or cause orbital inclinations need to be explained. The observed elliptical orbital motions as well as the free fall of bodies within the earthbased laboratories are simply the combined effect of the Keplerian rotation of the real space and the relatively very slow usual inertial motions of the planets and force free bodies with respect to this local moving and distorting real space. Auspiciously, besides disclosing the genuine gravitational physics, this spacedynamics also provides correctly the genuine physical origin of all the other observed effects, caused by the gravitational fields on the propagation of light and on the rate of clocks, including all the effects recently discovered with the help of the GPS as will be outlined in the coming sections. It also simulates Einstein's spacetime curvature and provides a simple solution to the problem of dark matter and dark energy. Dark matter and dark energy are actually the most flagrant and the most disturbing shortcomings of the current theories of space and gravitation.

\section{Recent Experimental Observations that Contradict the Fundamental Postulates of the Theory of Relativity}

\subsection{Observations that Invalidate Postulates of Special Relativity}

In Einstein's view, the only feasible method for measuring the velocity of light is by keeping the go-return roundtrip travel time of a light pulse along a known distance using a highly precise clock. However, absolutely all clocks count time in terms of a time standard, which may be the oscillation period of a stable classical or quantum oscillator. Evidently, such time standards (EM oscillations) are themselves go-return travel times of de Broglie matter waves along a certain distance that is entirely equivalent to the light go-return roundtrip. The quantized energy level structure of the oscillators (nuclei, atoms, molecules etc.) is well known to be sensitive to motions [4]. It seems obvious that the go-return of the light pulse and the go-return of the time standards are affected in the same proportion by motion of the laboratory with respect to a possible medium transmitting the matter waves (light). Hence, the constancy of the velocity of light, measured in this way, is obviously not a property of light but is a characteristic property (artifact) of the measuring method that simply cannot give a different result. Measuring the velocity of sound by an analogous method, using a "sound-echo-clock" too would lead to the erroneous conclusion that the velocity of sound is a universal constant. Moreover, in order to the go-return method to be valid at all, the velocity of the light pulse must be exactly the same in the go and in the return 
travel. Einstein justified this with base in the null results of Michelson light anisotropy experiments and the postulate of the perfect isotropy of light. However, as Einstein's interpretation of these null results was made without taking into account the true kinematical circumstances of the earthbased laboratories, this postulation has clearly not been made within a secure physical basis. Currently several reliable experimental observations prove the postulates of the intrinsic constancy and isotropy of light to be false $[3,5,6]$.

There are in fact two quite different categories of Michelson light anisotropy experiments. Most anisotropy experiments searched for light anisotropy along the orbital and or cosmic motion of Earth. Some others searched for light anisotropy with respect to the earthbased laboratory itself. The Michelson anisotropy experiments of the first category systematically found null anisotropy. In some of these experiments the interferometer did not rotate in the laboratory $[7,8]$ and so were intrinsically unable to detect anisotropy with respect to the laboratory, but obviously could detect anisotropy due to the orbital and cosmic motion of Earth. Several of these experiments using lasers or the Mössbauer Effect [9] gave null results because of recognized flaws in their conception [10]. The second category comprises few but among them the most sensitive interferometers known and practically all of them obtained small positive anisotropy of about $8 \mathrm{~km} / \mathrm{sec}$ [11-13]. Please see Section 10.1 for details. These experiments [12] gave evidence that this anisotropy is nearly constant the whole day and the whole year and has practically a fixed direction with respect to the earthbased laboratories. However, as these anisotropy data could not be explained in the epoch and moreover are nearly two orders of magnitude smaller than expected from the ether theories, they normally are imputed to spurious effects or simply ignored.

Recent unmistakable and reliable experimental and observational facts, most of them achieved with the help of the GPS, have a profound impact on the current world conception as well as on the experimental fundamentation of the postulates underlying the TR. Synchronization of the GPS clocks by the method of common view, consisting in redundant and iterative synchronization of the clocks on board of the 24 orbiting GPS satellites and the clocks within the earthbased stations round the world is attaining $0.1 \mathrm{~ns}$ (time for light to travel $3 \mathrm{~cm}$ ). With the help of these synchronized clocks, the one way velocity of electromagnetic (EM) signals (light) have been precisely measured. Many observations show that the transit time of EM signals (light) between satellites and or between satellites and earthbased stations depends on the velocity of the receiver analogously as for sound waves. Several observations reveal a clear discrepancy from the postulates of the theory of relativity $[3,5,6]$. This shows that the above assertions about non-null light anisotropy results of Michelson anisotropy experiments are not in isolation. They are corroborated by measurements of the one way velocity of light.

A full survey of the observations made with the help of the GPS goes beyond the scope of the present work. Here only the most relevant and clear-cut examples may be outlined. One such clear-cut observation is the one made in the Gravity Recovery and Climate Experiment (GRACE) [3]. In this experiment two satellites moved in the same sense in coplanar and practically identical polar orbits at $500 \mathrm{~km}$ of altitude, separated from each other by $\sim 200 \mathrm{~km}$ and moving with a velocity of nearly $8 \mathrm{~km} / \mathrm{sec}$. The positions of the satellites were monitored by the GPS within 0.03 meters. On exchanging signals between the two satellites, it was observed that the signal transit time from the leading satellite to the rear satellite corresponded to a shortening by more than 5 meters, while the signal transit from the rear satellite to the leading satellite was lengthened by more than 5 meters. These discrepancies correspond in both cases exactly to the distances moved by the receiving satellite during the signal transit time of the signals and are consistent with signal anisotropy of nearly $8 \mathrm{~km} / \mathrm{sec}$ with respect to the satellites. This anisotropy is two orders of magnitude larger than the experimental precision of the experiment and, although this anisotropy being much smaller than the known orbital and or cosmic velocity of our planet, it clearly and doubly invalidates the constancy and isotropy of the electromagnetic signal (light). The fact that the anisotropy effects correspond exactly to the distance moved by the receiving satellite in the two opposite directions excludes the possibility of the Lorentz contraction of distance. This light anisotropy along one way corroborates almost exactly the light anisotropies of about $8 \mathrm{~km} / \mathrm{sec}$ found with respect to the earthbased laboratories by the most sensitive Michelson experiments (see Section 10.1 for details). The fact that this anisotropy for the moving satellites along the polar orbits is of nearly $8 \mathrm{~km} / \mathrm{sec}$, closely the same found by the earthbased anisotropy experiments, is of the highest importance. It signals very clearly the true kinematical circumstances of the two satellites and of the earthbased laboratories with respect to the medium propagating the EM signals (light). It also clearly and specifically signals to the spacedynamics that gives origin to the inertial dynamics observed within the Earth's gravitational field.

Another observation that also clearly conflicts with the constancy and isotropy of the velocity of light was discovered during the implementation and calibration of set-ups for Very Long Baseline Interferometry (VLBI) radio astronomy observations. The resolution of optical and radio astronomy observations can be improved by orders of magnitude by analyzing the data recorded in 
different observatories over the earth surface using interferometric methods. The condition is that these data be synchronous. The method consists in superposing coherently the data recorded in different observatories with the help of computers taking into account the instantaneous position of the antennas etc. For the (VLBI) radio astronomy observations clock synchronization at intercontinental distances via the GPS achieve 0.1 ns. Nevertheless, on testing the so synchronized clocks by confronting them with the arrival of the wave fronts from distant pulsars, which according to the TR may be synchronous, it was observed that the pulsar signal reaches the foregoing side of Earth $4.2 \mu \mathrm{s}$ before the rear side along the orbital motion of Earth [5,6]. This discrepancy exceeds the time resolution by more than four orders of magnitude. Nevertheless along the transverse direction the arrival of the pulsar signal was synchronous. This apparent discrepancy in the GPS clock synchronization is again raising very hot debates about the nature of space. Some people [5] speak of scandalous clocks that are biased along the Earth's orbital motion, others [14] see in these facts definitive prove that the velocity of light along different directions within the solar system is not the same. In Section 10.8 the simple and genuine physical origin of this asynchrony in the arrival of the pulsar signal will be given. The fact that the arrival of the pulsar signal is out of synchrony along the Earth's orbital motion and is synchronous along the transverse direction is a very important and explicit hint to the spacedynamics that gives rise to the inertial dynamics within the solar gravitational field as will be disclosed in subsequent sections.

In the coming Sections it will become clear why the orbital and cosmic motion of Earth causes no light anisotropy and for what reason a definite small positive anisotropy along a horizontal direction of nearly $8 \mathrm{~km} / \mathrm{sec}$ the same with respect to all the earthbased laboratories round the world is observed and why an anisotropy of the same value is measured with respect to satellites moving along polar orbits. All these observational facts hint clearly at the true kinematical circumstance of respectively the planet Earth in the solar gravitational field and the earthbased laboratories with respect to the real space that propagates light. These kinematical circumstances will turn out to be exactly those giving rise to the inertial dynamics observed within the gravitational fields that are responsible for the non-inertial character of the earthbased laboratories. From this viewpoint, the conclusion that light is inherently constant and isotropic overlooks completely the observational facts and thus has no physical fundaments.

\subsection{Observations that Invalidate the Premises Underlying the General Theory of Relativity}

Ten years after the publication of the Special Relativity
(SR) Einstein repudiated the reality of the Newtonian gravitational forces and proposed that the gravitational pull is locally equivalent to an inertial force. From his viewpoint, the rules governing light and the motion of free bodies within gravitational fields have to be gauge invariant likewise they are in free space. This has lead to General Relativity (GR). It certainly was arduous for Einstein to put in practice the idea that the gravitational pull is equivalent to an inertial force because in his view space although flexible still has no active participation in the gravitational dynamics. He carefully adopted the abstract geometrical way according to which massive bodies cause time dilation and deform the metric of space in their neighborhood. Matter says the spacetime continuum how to curve and the curved spacetime says matter how to move. In the adequately curved spacetime, the path of light and the orbits of force-free bodies correspond to geodesic lines. This pretends to be a generalization of the principle of inertia and of Fermat's principle within the curved spacetime. Einstein emphatically rejected the idea of the objective reality of space. He often expressed his satisfaction in having succeeded to banish from his theory even the last trace of objective reality of space. However, how could the totally empty space (absolute nothingness) give support to geometry? What is the meaning of geometry of this absolute nothingness? Apparently Einstein had the conviction that the whole activity of the gravitational fields can be synthesized in terms of the hypothetical spacetime geometry. It however is a question of elementary logic that, if the spacetime curvature within gravitational fields is real so that it can effectively say matter how to move, then space that gives physical support to this curvature necessarily must be real too.

Many people believe that GR accounts for all the observed effects caused by gravitational fields. However, in reality GR is unable to explain an increasing number of clear observational facts, several of them discovered recently with the help of the GPS $[3,5,6]$. For instance, GR predicts the gravitational time dilation and the slowing of the rate of clocks by the gravitational potential of Earth, of the Sun, of the galaxy etc. Due to the gravitational time dilation of the solar gravitational potential, clocks in the GPS satellites having their orbital plane nearly parallel to the Earth-Sun axis should undergo a 12 hour period harmonic variation in their rate so that the difference between the delay accumulated along the half of the orbit closest to the Sun amounts up to about 24 ns in the time display, which would be recovered along the half of the orbit farthest from the Sun. Such an oscillation exceeds the resolution of the measurements by more than two orders of magnitude and, if present, would be very easily observed. Nevertheless, contradicting the predictions of GR, no sign of such oscillation is observed. This is the 
well known and so long unsolved non-midnight problem $[5,6]$. In fact observations show that the rate of the atomic clocks on Earth and in the 24 GPS satellites is ruled by only and exclusively the Earth's gravitational field and that effects of the solar gravitational potential are completely absent. Surprisingly and happily the GPS works better than expected from the TR.

Time dilation is well known to be caused by motion as evidenced by the increased lifetimes of speeding Muons and the redshift of the radiation emitted by speeding hydrogen atoms [4]. In the STR this time dilation effect is imputed to the relative velocity $\left(v_{r}\right)$ with respect to the observer. To first approximation this effect is proportional to $\left(v_{r}^{2} / c^{2}\right)$. Another time dilation effect predicted by GR is the gravitational time dilation, which to first approximation is linear in the gravitational potential $\left(U / c^{2}\right)$, where $U=\gamma M / r$ and $M$ is the mass of the gravitational source. In the Earth's gravitational field this effect has been evidenced by the atomic clocks on Earth and certainly may be observed for clocks fixed within the solar gravitational field too. Obviously the gravitational slowing of the atomic clocks on Earth cannot be due to relative velocity because these clocks rest with respect to the laboratory observer. What is immediately disturbing here is that two completely distinct physical causes produce identical effects, which by it alone is highly suspicious. GR gives only a geometrical interpretation to the gravitational time dilation. However, if motions cause time dilation, why then does the orbital motion of Earth suppress the time dilation caused by the solar gravitational potential on the earthbased and GPS clocks? Absurdly in one case motion causes time dilation and in the other case it suppresses it. This contradiction lets evident that what causes the gravitational time dilation is not the gravitational potential and that moreover this time dilation cannot be caused by a scalar quantity. If the time dilation shown by the atomic clocks within the earthbased laboratories is not due to the gravitational potential and cannot be due to relative velocity too then it is necessarily due to some other cause. This impasse once more puts in check the central idea of the TR, according to which the relative velocity with respect to the observer is the physical parameter that rules the effects of motions. The above facts show that the parameter that rules the effects of motions is not relative velocity but a velocity of a more fundamental nature.

The observed slowing of the atomic clocks, resting in the Earth's gravitational field, proportional to the Earth's gravitational potential must be related with the observed small constant anisotropy of light of nearly $8 \mathrm{~km} / \mathrm{sec}$ with respect to the earthbased laboratories. In fact these two effects are both proportional to closely $8^{2} / c^{2}$ where (c is in $\mathrm{km} / \mathrm{sec}$ ). Similarly the absence of effects due to the solar gravitational potential on the clocks orbiting (with
Earth) round the Sun must be related with the absence of light anisotropy due to the orbital motion of Earth as is well known. The only reasonable explanation acceptable for these observations is that the motion, which causes the small light anisotropy within the earthbased laboratories, is the same as the one that causes the observed small decrease of the clock rates on Earth and that the orbital motion of Earth, which suppresses the gravitational time dilation due to the solar gravitational field, also suppresses the light anisotropy due to the orbital motion of Earth. If the true cause of time dilation is motion as is demonstrated by Muons and atoms speeding at high velocity, then visibly the cause of both light anisotropy and the gravitational time dilation, observed within laboratories fixed within gravitational fields, is a mysterious implicit velocity $(V)$. This appoints velocity as the unified cause of time dilation. It is the usual velocity in the case of clocks speeding in free space and it is this implicit velocity in the case of clocks fixed within gravitational fields. It hence is velocity in a more fundamental sense (not the relative velocity) that rules time dilation and the spectral redshift of radiation sources fixed within a gravitational field as well as that of atoms speeding in free space [4]. This implicit velocity $V_{i}$ is what causes the gravitational time dilation and the small light anisotropy observed within the earthbased laboratories. The magnitude of the light anisotropy, of time dilation and of the spectral redshifts observed within laboratories fixed within the gravitational fields of Earth and of the Sun indicate that this implicit velocity is given by

$$
V_{i}=[\gamma M / r]^{1 / 2}
$$

where $\gamma$ is the gravitational constant, $M$ is the mass of the Sun and $r$ is the radius of the earth orbit. Interesting indeed $V_{i}^{2}=U=\gamma M / r$, which is the parameter that is well known to rule the gravitational time dilation. However, this implicit velocity is effective only within laboratories fixed within these gravitational fields. Within earthbased laboratories (neglect the very slow Earth's rotation) this velocity is nearly $8 \mathrm{~km} / \mathrm{sec}$ and for laboratories fixed within the solar gravitational field at the orbital distance of Earth it is $30 \mathrm{~km} / \mathrm{sec}$. Nevertheless, the absence of effects of the solar gravitational potential on the rate of clocks moving with Earth and the zero light anisotropy due to the orbital motion of Earth show that effects of the implicit velocity $\left(V_{i}\right)$ related to the solar gravitational field is set to zero by the orbital motion of Earth. This unambiguously proves that the orbital velocity of Earth subtracts from the implicit velocity $V_{i}$. The only way to rationalize these observational facts all together at once is admitting that the real space, the one that rules the propagation of light and the inertial motion of matter and is the ultimate reference for rest and for motions, is itself moving round the Earth in the sense of 
the Moon's orbital motion as well as round the Sun in the sense of the Earth's orbital motion according to a velocity $V=[\gamma M / r]^{1 / 2}$. While the observations, described earlier in this section, show that the postulates of the intrinsic constancy and isotropy of light are false, the observed absence of effects of the solar gravitational potential on the GPS clocks and the absence of light anisotropy due to the Earth's orbital motion provide positive and unmistakable prove that space that rules the propagation of light and the inertial motion of matter is moving round the Sun according to a velocity field closely consistent with the Earth's orbital motion.

Obviously to assume that Earth is kinematically privileged and is the only planet that is commoving with the real space is not reasonable. Hence, in this velocity field all the planets must be commoving with the real space. The only possibility is that this velocity field is the Keplerian velocity field:

$$
V(r)=[\gamma M / r]^{1 / 2}
$$

The $(1 / r)^{1 / 2}$ dependence of this velocity field of real space round a spherically symmetric mass must be a completely general feature. In the coming sections it will be shown that motion of space round the Sun and round all the matter concentrations throughout the universe, according to velocity fields closely consistent with the local main astronomical motions in these systems, correctly induces the inertial dynamics observed within the respective gravitational fields. The motions of bodies and light within gravitational fields will turn out to be simply the combined effect of the local motion of space and the inertial motion with respect to this moving space. Moreover, in Section 4 it will be shown that the simple free-fall of bodies, observed within the earthbased laboratories, corroborates exactly this same spacedynamics and independently paves a safe route to the genuine physical origin of gravitation. Please see in Sections 3 throughout Section 6 how this velocity field of the real space appropriately generates the whole inertial dynamics observed within the gravitational fields. See also in Sections 7 and 8 how this spacedynamics generates the galactic gravitational dynamics and the antigravitational interaction causing the accelerated expansion of the universe. See moreover in Section 9 the genuine physical origin of the light anisotropy, of the gravitational time dilation, of the spectral redshifts, of the non-Euclidean spacetime metric underlying Einstein's spacetime curvature and in Section 10 a large number of effects, predicted by spacedynamics, that get all a straightforward and genuine physical explanation. Specifically the reason for zero anisotropy of light due to the orbital motion of Earth and the local small constant anisotropy measured within the earthbased laboratories is discussed in detail in Section 10.1. The absence of effects of the solar gravita- tional field on the rate of clocks moving with Earth is discussed in Section 10.7.

It may be important to add here that the typical velocities in all of the conventional relativistic experiments are many orders of magnitude larger than the velocity of 8 $\mathrm{km} / \mathrm{sec}$ of the earthbased laboratories with respect to the real space. On the other hand, the experimental resolution of all these experiments, excepting the light anisotropy experiments, is several orders of magnitude too low to evidence effects of this low implicit velocity. Hence, these so called relativistic effects can easily be reinterpreted as effects caused by the velocity with respect to the real space.

The time dilation effects predicted by the TR have been claimed to be confirmed by round the world Sagnac like experiments. In the Hafele and Keating experiment [15] atomic clocks were transported by commercial jets westward and eastward round the Earth. Due to the rather erratic space and time path and the instability of the clocks much larger than the effect to be measured, the results obtained in these precarious experimental circumstances are in no way reliable. Moreover, the authors have completely omitted the effects of the velocity of the $\mathrm{Cs}$ atoms in the Cs beam atomic clocks that is comparable with the average flight velocity of the jets. Another round the world Sagnac experiment by Allan, Weiss and Ashby [16] was made by parts, involving three GPS satellites and three earthbased stations. As the satellites moved at more than $2 \times 10^{4} \mathrm{~km}$ of altitude, the signal path is mostly radial and aberration as well as anisotropic velocity effects cannot be neglected. Moreover the idealized platform of the three involved GPS satellites rotates on average two times faster than the platform of the earthbased stations and therefore, from the viewpoint of the involved GPS satellites, Earth rotates due West and the Sagnac path loop continually deforms. Both these round the world Sagnac experiments were performed within exceedingly precarious experimental conditions and therefore can hardly provide trustworthy conclusions. On the other hand, the time dilation effect of the solar gravitational field on the atomic clocks orbiting with Earth round the Sun, which is predicted by GR but not observed, is a highly precise observation. It exceeds by orders of magnitude the experimental precision and hence is infinitely more reliable. If the orbital motion of Earth round the Sun suppresses the time dilation due to the solar gravitational field and moreover does not show the predicted relativistic time dilation due to this orbital motion, then it seems reasonable that a clock in a satellite orbiting round the Earth in a direct equatorial orbit or in a jet flying round the Earth too should give no evidence of such a relativistic time dilation. The relativistic time dilation alleged in both these round the world Sagnac experiments is in clear and frontal contradiction with the 
absence of such a relativistic time dilation effect in the case of the orbiting Earth round the Sun.

The spacetime curvature of GR predicts correctly the excess time-delay of radar signals in go-return roundtrips within the solar system (Shapiro effect) as well as the gravitational deflection of light by gravitational fields. It however is unable to explain why the arrival of the signal from distant pulsars to the earthbased observatories, synchronized to within $0.1 \mathrm{~ns}$ with the help of the GPS, is out of synchrony up to $4.2 \mu \mathrm{s}$ in the direction of the orbital motion of Earth $[5,6]$, while being synchronous in the transverse direction. Unfortunately the velocity of light as well as the excess time delay (Shapiro effect) and all analogous experiments within gravitational fields have not been measured in one way travels but only in two way roundtrips. Therefore such experiments can provide only the average velocity of light and the effective excess time delay over the full roundtrip and hence they hide all the involved anisotropies. Please see the true origin of all these effects in Section 9. Specifically the origin of the Shapiro effect is discussed and calculated in Sections 10.3 and the gravitational light deflection is quoted in Section 10.10. The fact that the pulsar signal reaches first the antenna located at the foregoing side of Earth and only $4.2 \mu$ s later the rear antenna along the Earth's orbital motion and is synchronous for antennas along the transverse direction provides a very specific hint to decipher the spacedynamics underlying the gravitational mechanism. Please see the genuine physical origin of this observation in Section 10.8.

Astronomical observations also show plenty of red/ blue shifts of non-Doppler origin that are especially clear-cut in binary stars and binary galaxies. These shifts achieve values up to several orders of magnitude too large to be explained by GR [17]. Besides these observations are the Pioneer 6 unexplained frequency shifts $[17,18]$. Please see Section 10.4 for the simple and genuine physical origin of the red/blue shifts caused by variable time delay of light going across moving gravitational fields. Another puzzle to which GR gives no answer too is the incredible order (disk shape) of the astronomical motions in our solar system, in our galaxy and in most other galaxies. In the literature, the reason for this remarkable order usually is imputed to a final equilibrium state that has evolved (relaxed) from an initially rather random state. However, according to Section 10.9, this order has a very simple physical origin that is dictated by the gravitational mechanism itself. This order simply minimizes the velocity of matter (astronomical bodies) with respect to the moving real space. GR also cannot explain the small light anisotropy of about $8 \mathrm{~km} / \mathrm{sec}$ along a fixed direction with respect to the earthbased laboratories, which has been detected by the highly sensitive rotating Michelson experiments and has recently been corroborated by measurements of the one way light velocity along the North-South direction [3]. All these effects hint clearly to the genuine physical origin of the inertial dynamics, observed within the gravitational fields, to be disclosed in the coming sections. Certainly the most emphatic evidence that the current view about the gravitational physics is wrong is provided by the need of postulating a preposterous amount of mysterious dark matter and dark energy to explain the observed galactic gravitational dynamics and the accelerating expansion of the universe.

In the totally empty space of the TR, the only conceivable interaction between remote bodies could be the quantum exchange interaction. Quantum Gravity (QG) is trying to implement this in terms of gravitational forces created by the quantum exchange interaction mediated by virtual gravitons. However, the experimental observations are not giving support to the predictions of QG. While the Standard Elementary Particle Model has been extremely successful in describing the makeup and the interactions between the elementary particles, QG is now struggling for nearly four decades without any success. What we really have actually are two theoretical supermodels the purpose of which is explaining the observations respectively in the microcosm and in the macrocosm, but are incompatible with each other. This means that one of them is fated to disappear. While the quantum theory explains the interaction between elementary particles in terms of forces created by the quantum exchange interaction, GR denies the existence of gravitational forces and imputes gravitation to the geodesic (inertial) motion within the curved spacetime.

No doubt that Einstein's local equivalence of the gravitational and inertial effects is a precious legacy that provides us with the key to correctly understand the gravitational physics. However, the geodesic motion in the curved spacetime is only an abstract (fictitious) law of motion. Although it is able to correctly predict many effects of the gravitational fields, it does not bring to light the true physics causing these effects. This disables it to explain an increasing number of consistent observations.

In the sections coming hereafter a new conception of space, matter and gravitation, fully committed to reality, will be outlined that solves all at once the troubles with gravitation that are afflicting the current theories. This new conception does not only provide the genuine physical origin of the inertial (gravitational) dynamics observed within the gravitational fields but also correctly accounts in terms of genuine physical effects for all the other observed effects caused by the gravitational fields.

\section{The Quantum Fluid Nature of Space}

Likewise Ptolemy's guess that Earth is fixed on a firm 
basis encumbered comprehension of the astronomical motions in the past, so does the guessed static space encumber now our comprehension of the gravitational physics. The nature of space must not be guessed but learned from extensive and judicious examination of the experimental facts. The concept of empty space has evolved quite a lot since the creation of the TR. The vacuum fluctuations are known to cause observable effects in the energy levels of the Hydrogen atom known as the Lamb shift [19] or even mechanical effects like the Casimir effect [20]. However, what exactly is fluctuating in the quantum vacuum and what exactly oscillates in the de Broglie matter waves? Frequent answers to such questions in the literature affirm that wave functions are only a mathematical trick that these oscillations and fluctuations cannot individually be observed and therefore must not be seen as real. However, statistically these oscillations become quite evident and are commonly observed. For instance, in Quantum Electrodynamics, the electromagnetic waves and lasers are conceived as the coherent superposition of such statistical oscillations. It seems that the true reason for dodging genuine answers to such questions visibly is the fact that this would imply bestowing to empty space (vacuum) some kind of objective reality, which is incompatible with the principle of relativity. In the present work all this activity will be delegated to the real quantum space itself that may be the all pervading Higgs boson condensate or something similar. The quantum vacuum is to be seen simply as the ground state of the quantum space. Besides the microscopic quantum activity, giving rise to the properties of the elementary particles, there also is intense macroscopic activity giving rise to the gravitational fields and the inertial dynamics observed within these gravitational fields.

The main goal of the present work is to show that motion of the real space (the same space that rules the propagation of light and the inertial motions of matter) in the ordinary three dimensions round each matter concentration throughout the universe, according to a Keplerian velocity field consistent with the local main natural astronomical motions, appropriately engenders the inertial dynamics (gravitation) of bodies exactly as observed within the gravitational fields. To say that this real space is moving in the ordinary three dimensions, round an astronomical body like Earth, consistently with astronomical (natural satellite) motions, means that a particle that rests with respect to this moving space is carried around without the need of any forces and without the need of moving by itself. This Keplerian velocity field closely consistent with the astronomical motions is exactly what the null results of the Michelson light anisotropy experiments demand. From the viewpoint of this spacedynamics, stars in the galaxy, the planets in the solar system as well as their satellites do all closely rest with respect to the real and moving space. Einstein abhorred the idea that Earth rests with respect to the static ether or the static space because in his view this would privilege Earth in detriment to the entire remainder universe. However, in the above velocity field of the real space, the astronomical bodies throughout the universe are all equally privileged, which means that none is favored. On the other hand, a particle that is not moving exactly the same way as the real space necessarily is moving with respect to real space. This motion with respect to the moving and distorting real space is subject to refraction effects resulting in non-circular or non-equatorial orbits. In the coming sections, it will be shown that this velocity field of the real space, besides naturally and appropriately generating the inertial dynamics (orbital motions and free-fall) observed within the gravitational fields, forthrightly generates in terms of well known and genuine physical effects all the other effects, caused by the gravitational fields on the velocity of light and the rate of clocks. The spacedynamic origin of gravitation has been stressed by the author in two previous publications [21,22]. However, this new article aggregates plenty of new evidence.

In order to this spacedynamic gravitational physics become effective, it is necessary that matter and fields (light) be connected with the real and moving space likewise the usual mechanical waves are connected with their medium of propagation. This means that the moving real space (vacuum) must be locally the ultimate and absolute reference for the motions of light and particles. This requisite is automatically accomplished if the real space (vacuum) is a primordial and physically real background medium in which all the fields and elementary particles are excited analogously as are the ripples in the ocean water or sound noise in the atmosphere. Many experimental observations prove that motion of elementary particles and fields are associated with the de Broglie matter waves. Hence, if this space moves and deforms, the matter waves are moved and deformed in the same way. The de Broglie waves are dragged and refracted analogously as sound waves are by wind gradients [23-25]. In this dynamical real space, the curved motion of the force-free bodies in gravitational fields too is an inertial (forceless) motion likewise they are in General Relativity (GR). However, here the very slow inertial motions are with respect to the real space while it moves in the ordinary three dimensions and not with respect to the static curved space. The curved motions of the astronomical bodies within gravitational fields are simply the combined effect of the circulation of the real space about the gravitational center and of the inertial motion of these bodies with respect to the locally moving and distorting real space. It hence is clear that Einstein was right in equalizing locally the gravitational pull to an inertial 
force. However, unfortunately he misinterpreted the null results of the Michelson light anisotropy experiments, which has misled him to an incorrect concept of space, precluding the possibility of putting in practice the principle of equivalence in a physically realistic way and identifying the gravitational pull as a usual genuine inertial effect.

If the motion of real space is consistent with the local main astronomical motions, then planets of the solar system, the stars in the galaxy etc. are motionless with respect to it. Bodies in direct circular equatorial orbital motions are naturally in a state of physical rest with respect to real space that propagates light and therefore the light velocity is isotropic with respect to these bodies. Hence, analogously as the rotation of Earth exempted people from explaining the diurnal transit of the heavens, so does this spacedynamics exempt us from explaining the circular orbital motions of the planets round the Sun. Only the behavior of bodies, which are not moving in exactly the same way as the real space, needs to be explained. Such bodies are moving according to the usual principle of inertia with respect to the real and moving space. Besides engendering correctly the inertial dynamics observed within the gravitational fields, this spacedynamics forthrightly resolves the century old light isotropy/anisotropy puzzle. It also will be seen to explain forthrightly in terms of genuine physical effects (see Sections 9 and 10) the observed small light anisotropies, the gravitational slowing of the clocks and the gravitational spectral redshifts. Spacedynamics simulates the nonEuclidean metric underlying Einstein's spacetime curvature and correctly causes the excess time delay in goreturn roundtrips of radar signals within the solar system. It also straightforwardly explains in terms of genuine physical effects all the recently discovered observational facts made with the help of the GPS (see Section 10).

The idea that the real space is moving in the ordinary three dimensions may seem far-fetched and frightening likewise the earthglobe floating in space without a firm basis seemed in the days of Copernicus and Galileo. However, this fear is only the manifestation of our neophobia. From the epistemological point of view, a real and moving space is per-se at least as reasonable as the Newtonian static space or the flexible space of relativity. Why would a static space be more reasonable? What would keep it up static? If it can curve, why could it not be moving? Nothing, absolutely nothing can pre-establish that space is so or so. The nature of space must be discovered and not guessed. Only experimental facts and judicious observations can say if space is static or dynamic, real or simply a false background contrived by our mind. Space is simply what it must be in order to be able to play the role that we see that it is playing. We see that it plays the role of a primordial and dynamical back- ground of the material universe. This is the hint and it is better not to ignore it. Obviously the real space that is the ultimate support of the material phenomena and the ultimate reference for motions of matter and fields must be something very strong and powerful. In the subsequent paragraphs the possible nature of space will be outlined.

Clearly no classical medium has the necessary properties to play the role that is being imputed to the real space. Only a quantum fluid like real space comes into consideration. Quantum fluids, besides deformable and perfectly inviscid for steady motions, possess a peculiar dynamical rigidity that enables them to actively resist against changes of their actual state of motion. Usual quantum fluids like superfluid helium and superconducting states are Bose-Einstein (BE) condensates of bosonic or bosonic like particles. The particles of a $\mathrm{BE}$ condensate are in a collective state and hence are indistinguishable and the position of individual particles is undetermined. Therefore motion of quantum fluids cannot be conceived like classical motion, but can only be described in terms of the amplitude and phase of a complex order parameter [ $\Psi=\psi(r) e^{i \varphi}$, where $\varphi$ is the phase]. While a constant phase difference between two fixed points in space corresponds to uniform motion of the quantum fluid, a phase difference changing with time corresponds to acceleration. It is well known that phase correlation of the $\Psi$ between the volume elements of a quantum fluid does not act in the sense of stabilizing the position of one volume element with respect to the others (solidification) but in the sense of preventing changes in the actual state of motion of the quantum fluid throughout. This correlation between the volume elements provides the quantum fluid with the ability of actively resisting against changes of the state of motion, which is fundamentally different from the usual passive inertia. This active resistance against changes is responsible for the perfect persistence of the local motions (absence of internal dissipation). It also confers to the quantum fluid a dynamical stiffness that enables it to transmit perturbations at very high velocity even flowing and deforming like a usual fluid. Therefore, any spatial variation of the amplitude and or phase of $\Psi$ costs energy and the steeper this variation is the more energy it involves as described by the Schrödinger equation [26] and the Ginsburg-Landau equation [27]. On the other hand, phase coherence minimizes this energy of the perturbation. Local excitations, consisting of stable circulation fields along closed loops, contain a locked-in phase displacement. Single-valuedness and phase coherence along these loops require that the phase displacements round the loops be an integer number $n$ of $2 \pi$, which leads to the intrinsic quantization. The origin of this intrinsic quantization is fundamentally different from the usual quantization due to particle confinement. Quasiparticles 
like Rotons, Maxons and Vortices in superfluid helium [28] as well as supercurrent vortices in superconductors [29] are well known examples of such stable and intrinsically quantized excitations.

Usual Bose-Einstein (BE) condensates are all very frail. Contrarily the quantum fluid like real space or quantum space $(\mathrm{QS})$ or quantum vacuum must be stable up to extremely high temperatures. Hence, if the QS is a $\mathrm{BE}$ condensate, it must be one of elementary bosons with a very high $\mathrm{BE}$ condensation energy, which means it must be stable up to very high temperatures. BE condensates with such high condensation energy is not a farfetched idea. The Standard Elementary Particle Model (SEPM) relies on the existence of such condensates. For instance, the all pervading Higgs condensate that gives mass to the elementary particles by the Higgs mechanism is believed to be stable up to about $10^{15}$ degrees Kelvin [30], the critical temperature of the weak nuclear interaction. The Higgs mechanism (Yukawa like exchange interaction plus spontaneous gauge symmetry breaking) was conceived to explain why the $W^{+}, W$ and the $Z$ gauge bosons of the weak nuclear interaction have mass. According to the SEPM, quarks and leptons interact with this Higgs condensate by the Higgs mechanism to get mass. The Higgs condensate has remarkable similarities with the superconducting condensate in metallic superconductors. It is to the weak and strong nuclear fields what superconductivity is to the magnetic field. While the superconducting condensate excludes magnetic fields from the superconductor, the Higgs condensate excludes the weak and strong fields from the vacuum. The exact constitution of the Higgs condensate is not settled to now. Besides the Higgs condensate, other very stable condensates too are predicted to exist. For instance, in Quantum Chromodynamics the color superconducting quark phases are predicted to be stable up the $10^{12}$ degrees Kelvin [31].

The idea of the QS being a primordial quantum fluid that has little to do with $\mathrm{BE}$ condensation and or with temperature is not a far-fetched idea too. What is essential to the spacedynamics gravitational mechanism is that the moving QS represent locally the ultimate reference for rest and for motion of matter and fields. This is accomplished automatically if the QS is the substrate in which all the elementary particles and fields are excited and in which they propagate according to the rules dictated by quantum mechanics or move classically according to the principle of inertia. If the Higgs condensate is indeed to play the role of the QS of spacedynamics, it must do much more than simply giving mass to the elementary particles by the Higgs mechanism. It must be the ultimate physical substrate in which all the elementary particles are excited and in which they propagate as persistent perturbations according to the law of inertia. It must be locally the ultimate reference for rest and for motion of mater and fields so that, on moving itself, it carries together with it this ultimate reference. The Higgs condensate also must provide the elementary particles with all the respective quantized physical parameters. Hence, besides the Higgs mechanism giving the elementary particles mass, other mechanisms must give them quantized electric charge, quantized intrinsic angular momentum, quantized gravitational mass etc. Hence, motion of matter with respect to the resting Higgs condensate or the motion of the Higgs condensate through resting matter produces exactly the same effects. If the Higgs condensate accomplishes this, it is able to move according to the Keplerian velocity field creating the gravitational fields.

The Standard Elementary Particle Model characterizes the various families of the elementary particles and their interactions in great detail. We know empirically that mass, charge, intrinsic angular momenta etc. of the elementary particles are intrinsically quantized and conserved. We know well the conservation laws and the quantization rules. Symmetry and super-symmetry too are fundamental guide lines in high energy physics. Nonetheless, the ultimate reason of the intrinsic quantization and of the laws of conservation is a mystery. A quantum fluid like space provides all the physical grounds for conservation and intrinsic quantization of its excitations. It also is a perfectly conservative and an ideal storage for momenta and energy in the form of local persistent and intrinsically quantized dynamical states. The quantum fluid like space has not only all the adequate mechanical properties but also possesses the whole infrastructure necessary to play the role of the physical background of the observed material phenomena. Within this view, the different elementary particles (rest mass $\neq$ 0 ) must be seen as the members of the quantized excitation spectrum of the QS itself. Hence, the elementary particles of a same class (electrons or protons etc.) correspond to exactly the same type of excitation throughout the universe. This explains why they are all so identical throughout the universe, one of the most remarkable and stupendous facts of nature. Fluctuations of the QS (quantum vacuum) also naturally may create the zoo of the numberless virtual particles.

However, what are the elementary particles made of? From the viewpoint of spacedynamics they are not autonomous self-existing entities somehow coexisting as strange bodies within the QS. They are essentially propagating spacedynamic perturbations. This means that they are made of pure spacedynamics. They are conceived as local persistent cyclic dynamical sates of the QS itself stabilized and quantized by locked-in phase displacements along closed loops, which is reminiscent of the quantized vibrations of superstring loops in superstring loop theory. According to some Super-symmetric 
Standard Models the Higgs field (condensate) has several components. Hence, if the Higgs condensate plays the role of the QS of spacedynamics, many different kinds of local dynamical states are possible, a specific one for each type of elementary particle. Persistence of the coherent motions of the QS also leads directly to the persistence of the motion of the particles, which means inertial mass and the inertial behavior of the particles and hence to conservation of the linear and angular momentum of all particles. Motion of particles is well known to be ruled by the propagation of de Broglie matter waves, which means that motion of these particles is effectively ruled by laws of propagation in the QS. Hence, the QS is effectively the ultimate reference for their motion. Therefore, "motion of the laboratory with respect to the resting QS or motion of the QS through the resting laboratory are perfectly equivalent and give rise to exactly the same physical effects."

In short, gravitational fields are created by velocity fields of the QS and the natural astronomical motions throughout the universe are basically the motion of the real QS itself. The free fall of bodies in the earthbased laboratories and the motion of bodies and the propagation of light along curved paths, observed within the gravitational fields, are simply the combined effect of the circular motion of the QS and the inertial motion of matter (propagation) with respect to the moving QS. All the other effects caused by gravitational fields are the simple and exact consequences of this same velocity field of the QS. The velocity of light is well determined with respect to the moving QS. Hence, measuring distances in terms of the go-return roundtrip time of light within a gravitational field without taking into account this effective velocity of light simulates the non-Euclidian metric underlying Einstein's spacetime curvature. The moving QS is the ultimate reference for the motions of matter and hence changing locally the velocity of the QS does not affect the material phenomena within a laboratory commoving with the QS. Motion of the QS is a typical gauge invariant. Viewed that all the astronomical bodies very closely rest with respect to the local QS, the physics that rules the material phenomena on Earth certainly is closely the same on the other planets and on all the astronomical bodies throughout the universe.

Summary of the evolution of our world concept: Before Copernicus and Galileo, Earth was seen as fixed on a firm basis. So people had to invent phantastic stories to explain the diurnal transit of the Sun, of the Moon and of the stars as well as their monthly and annual variations. Copernicus and Galileo introduced the heliocentric system and the rotation of Earth. So there was no need anymore to explain the diurnal transit of the heavens. Only the small monthly, annual and longer period variations needed to be explained. Newton adopted the helio- centric conception. He however considered the astronomical motions within a static and absolute Euclidean space. Hence, he had to invent the fictitious gravitational forces to account for the gravitational pull on objects and the orbital motion of the planets and satellites. Einstein (1916) refuted the reality of the Newtonian gravitational forces and asserted the local equivalence of gravitational and inertial effects. However, in his view, space although flexible still was inert. So he had to invent the spacetime curvature (the geometry of nothingness) to explain the free fall and the curved motion of bodies within gravitational fields.

In the present work, the concept of the real dynamical quantum fluid like space (QS) is introduced. This QS that may have to do with the Higgs condensate is the physical support of all particles and fields and hence the ultimate reference for their motions. This QS is neither static nor curved, but literally is moving in the ordinary three dimensions according to a velocity field consistent with the local main astronomical motions round each matter concentration throughout the universe. The astronomical motions can be observed thanks to the enormous velocity of light compared with the slow velocity of the astronomical bodies and of the QS. If the planets of the solar system likewise all other astronomical bodies are closely commoving with the QS, we need to invent nothing to explain their motion round the Sun or that of the Moon round the Earth because it is space itself that so moves and carries them around. The observed astronomical motions are essentially the motion of the QS itself combined with the very slow inertial motion (propagation) of these bodies with respect to the moving QS. Hence, likewise the rotation of Earth exempted people from explaining the diurnal transit of the heavens, so does this spacedynamics exempt us from explaining the orbital motion of the planets round the Sun. Only the small effects that distort the regular circular orbits into elliptic, parabolic (free fall) or eventually hyperbolic paths still need to be explained. Moreover, if the astronomical bodies very closely rest with respect to the moving QS that propagates light, it obviously is expected that the Michelson light anisotropy experiments searching for effects of the orbital and cosmic motion of Earth give a null result. This spacedynamics will be seen to account straightforwardly in terms of genuine physical effects for all the other observed effects, caused by the gravitational fields. This spacedynamics is, since Copernicus and Galileo, the first profound innovation in the view about the nature of space and, as will be shown, resolves all at once the troubles encountered by the current gravitational theories. Within this view Einstein's hypothetical spacetime curvature is only one more consequence of spacedynamics and arises because of the alleged intrinsic constancy and isotropy of light and the inappropriate go-return measur- 
ing method for distances in terms of this velocity of light.

\section{Origin of the Inertial Dynamics Observed within the Gravitational Fields}

In order to get mathematical rigor into the description of the velocity field that creates the gravitational field round a spherically symmetric mass, like Earth, let us define a system of non-rotating rectangular coordinate axes (XYZ) having origin fixed to the gravitational center and the $Z$ axis pointing due North along the rotation axis. Let $(r, \theta, \phi)$ be the respective usual spherical coordinates and $\left(\boldsymbol{e}_{r}, \boldsymbol{e}_{\theta}, \boldsymbol{e}_{\phi}\right)$ be the respective unit vectors along the $r, \theta$ and $\phi$ coordinates.

Besides the evidence that real space is moving from the observed light anisotropies and from the gravitational time dilation effects, discussed in Section II, other commonly observed facts in our daily life too give unmistakable evidence that real quantum space (QS) itself is moving in the ordinary three dimensions. In the introduction it has been asserted that the fact that free bodies within the apparently resting earthbased laboratories accelerate downward at $9.8 \mathrm{~m} / \mathrm{sec}^{2}$, although no force is acting on them, implies that these laboratories are not inertial references, that they are accelerating upward 9.8 $\mathrm{m} / \mathrm{sec}^{2}$. However, as these laboratories go on resting, it has been concluded that this upward acceleration can only be centripetal acceleration because only centripetal accelerations act under zero velocity along their instantaneous direction. Hence, the apparently resting earthbased laboratories must be moving within the local inertial references round axes located vertically above each laboratory (overhead). This can make a sense only if the local inertial references (local-IRs) are themselves rotating oppositely at the same rate about the same overhead axes so that the earthbased laboratories are left in apparent rest however under upward centripetal forces from the earthsurface. On one hand, this will say that at a given point only one unique rotating coordinate system can be the locally valid inertial reference and, on the other hand, that all coordinate systems not rotating exactly the same rate about the same overhead axis are not inertial references.

Due to the spherical symmetry of the gravitational field and the decrease of gravitation with altitude any such local IR can be valid at only one geometrical point and each local IR rotates necessarily about a different axis located overhead. These axes lie all along $\boldsymbol{e}_{\theta}$. This exclusively local validity of the IRs demands for completely new physics. A particle that moves with respect to the $X Y Z$ axes goes immediately through a continuous (infinite) sequence of different local inertial references that are rotating at different rates about different overhead axes. Obviously the particle moves within each local-IR according to the law of inertia. However, as the
local-IR as well as the non-inertial laboratory reference change from point to point, the usual description with respect to an inertial reference is not practical. Only the dynamics of a particle that rests at a fixed position with respect to the non-rotating $X Y Z$ coordinate axes can be described in a simple way from the viewpoint of the local-IR. If the particle is free to move, only the instantaneous inertial behavior with respect to the local-IR where it happens to be is defined. In view of these harsh difficulties and in addition the discovery that the rotation of the local-IRs is not a simple trigonometric rotation but a hyperbolic rotation, the inertial dynamics of bodies within the gravitational fields will be described in terms of the propagation and refraction of the de Broglie matter waves that are well known to rule the motion of particles and light. This description is much more appropriate in many aspects.

The only possible way to legitimate the upward centripetal acceleration of the earthbased laboratories and the rotation of the local IRs about an overhead axis, is admitting that space, the same space that rules the propagation of light and the inertial motion of matter, moves locally according to a velocity field that corresponds within each small volume element to the rotation rate of the local IR about the corresponding axis overhead. Obviously space cannot simultaneously rotate likewise a rigid body around the countless different overhead axes to reproduce the downward centrifugal effect within each infinitesimal volume element in accord with the spherically symmetric gravitational field of Earth. However, if the QS can move and deform like a fluid, it may be able to move according to a differential velocity field in which the velocity increases with decreasing distance from the gravitational center and engendering locally the velocity distribution within each infinitesimal volume element that corresponds to the rotation rate of the local IR about an overhead axis. This brings us back to the conclusions in the previous Section 2.2 where it has been shown that the absence of effects of the solar gravitational field on the rate of the GPS clocks as well as all clocks moving with Earth and the absence of light anisotropy due to the orbital motion of Earth lead unambiguously to the conclusion that real space moves round the Sun and round all other astronomical bodies according to a Keplerian velocity field consistent with the local main astronomical motions $V(r)=[\gamma M / r]^{1 / 2}$.

In the Keplerian velocity field the distribution of velocities within each infinitesimal volume element (please see Figure 1) corresponds to rotation round an axis overhead (the exact position of which will be determined) at each point within the gravitational field of Earth. The local rotation about an overhead (external) axis can arise only within a velocity field in which the velocity increases for decreasing $r$, that is, has a velocity gradient 
pointing toward the gravitational center, which is absolutely the case of the Keplerian rotation field.

Close to the equatorial plane the velocity distribution within each small volume element centered at $\left(r, \theta=90^{\circ}\right.$, $\phi)$ corresponds to rotation of the QS round an axis at $(r+$ $\left.r^{\prime}, \theta=90^{\circ}, \phi\right)$ that lies along $\boldsymbol{e}_{\theta}$ vertically above the volume element. Consider now a very large file of such infinitesimal volume elements along the equatorial line round Earth separated from each other by infinitesimal distances. Although the velocity distribution of the QS within each infinitesimal volume element corresponds to rotation about a different overhead axis, on letting the distance between neighboring volume elements tend to zero and connecting the flow lines of the QS through all these infinitesimal volume elements, the flow also corresponds to a circulation field round the $Z$ axis (please see Figure 2). Note that the positions of the overhead axes too run along a larger circular line above the equator round the Earth. Consider moreover that the motion of particles and light is ruled by the de Broglie waves and that the propagation of these waves is normal to their two-dimensional wave fronts. Each wave front supposedly propagates at the same velocity at all its points with respect to the QS. A particle that rests with respect to the $(X Y Z)$ axes within the velocity (gravitational) field necessarily is propagating with the implicit velocity $\boldsymbol{V}(r)=-[\gamma M / r]^{1 / 2} \boldsymbol{e}_{\phi}$ (against the velocity of the QS). Hence the corresponding de Broglie wave fronts are in the $[r, \theta]$ plane, that is, are perpendicular to the velocity field and hence are dragged backward by the velocity field of the QS at a rate that is larger at the lower side than at the upper side and therefore the wave fronts are slanted so that the instantaneous horizontal $(\phi)$ propagation velocity component is refracted downward at a rate corresponding to the rotation rate of the local-IR.

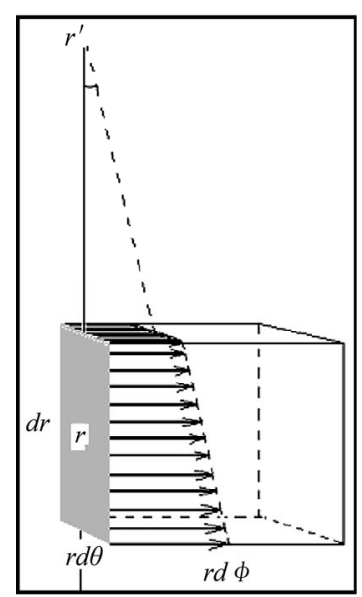

Figure 1. You are looking due north. The velocity field pointing along the $+\phi$ spherical coordinate within a volume element $r^{2} \mathrm{~d} r \mathrm{~d} \theta \mathrm{d} \phi$, consistently with a rotation about an overhead axis at $\boldsymbol{r}^{\prime}$, to be determined.

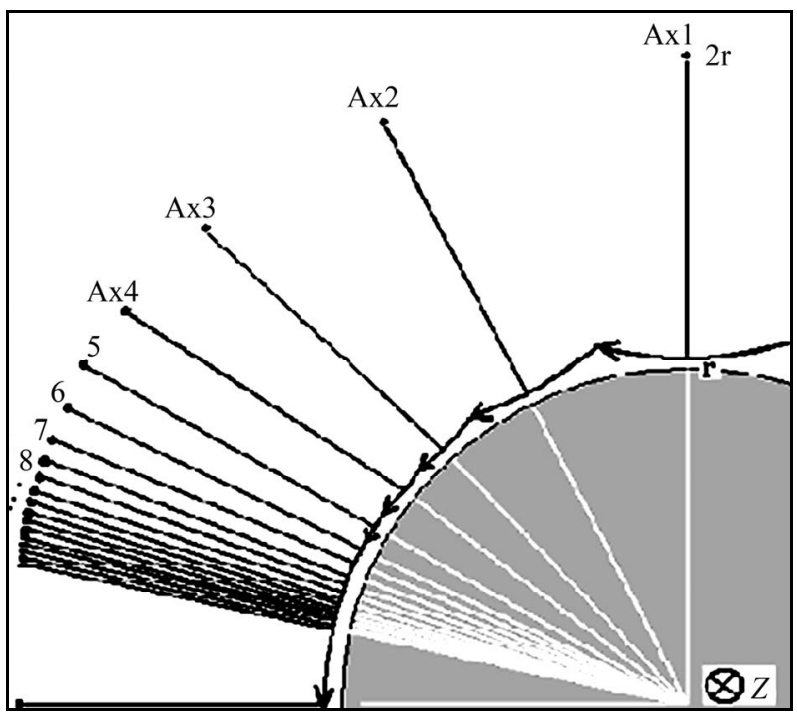

Figure 2. You are looking due south. The rotation of the QS about projected overhead axes at points $A_{1}, A_{2}, A_{3} \ldots$ within a file of numerous infinitesimally small volume elements along the equator. In the measure the distances between the volume elements become smaller and smaller, the rotation of the QS about the overhead axes and the corresponding circulation through the volume elements approximates more and more to a circulation field about the earth center. In the limit of infinitesimal distances between the points, the circulation of the QS is exactly a continuous circulation about the $Z$ axis because the rotation axes for the different volume elements too displace them along a circular line above the equator. If the Keplerian velocity field is spherically symmetric, this goes on valid on going away from the equator toward the poles.

However, the wave fronts of a particle that has only a velocity along the $r$ coordinate are in the $[\theta, \phi]$ plane and hence they cannot be affected by the rotation round the overhead axis. Besides this, stretching or compression of the wave length of the de Broglie waves affects the velocity of the particles according to the equation $p=h / \lambda$ and must be considered too. In the next Section 5 it will be shown that this radial velocity component is refracted in a sense opposite to that of the $\phi$ component at a rate proportional to $\mathrm{d} V / \mathrm{d} r$. Therefore the rotation of the local inertial references and the refraction of the de Broglie waves are not a usual trigonometric rotation, but a hyperbolic rotation as will be shown.

In flow fields, in which the flow velocity increases with increasing distance from the rotation axis, the radial and the tangential velocity components of a particle refract in the same sense, however not necessarily at the same rate. This constitutes a trigonometric rotation. In flow fields in which the flow velocity is constant with distance from the axis no refraction at all takes place. However, in flow fields in which the flow velocity increases with decreasing radial distance the radial and tangential velocity components of a particle with respect 
to the QS refract in opposite senses and constitute a hyperbolic rotation. An analogous behavior is well known to take place in the propagation of sound waves within whirl wind and within water flow gradients [23-25].

In order to the Keplerian velocity field of the QS $\boldsymbol{V}(r)=[\gamma M / r]^{1 / 2} \boldsymbol{e}_{\phi}$ to generate the right downward gravitational acceleration at all places round Earth, from the equator to the poles, it is sufficient that the magnitude of the velocity in the cylindrical velocity field be spherically symmetric about the gravitational center, that is, for a given $r$ be the same for all $\theta$ and $\phi$. This apparently is a universal characteristic of the velocity fields of the QS of all spherically symmetric gravitational sources. Figure 3 depicts the velocity of the QS $[V(r)]$ due to a homogeneous spherically symmetric distribution of mass as a function of $r$ along one radial line. For a spherically symmetric Keplerian velocity field this velocity function $V(r)$, for a given $r$, is the same for all $\theta$ and $\phi$. Therefore the distribution of velocity of the QS within small volume elements corresponds to a rotation about an axis vertically above the volume element at all places round the world, from the equator to the poles. In fact for the spacedynamic gravitational mechanism only the magnitude of the velocity in the velocity field

$$
\boldsymbol{V}(r)=[\gamma M / r]^{1 / 2} \boldsymbol{e}_{\phi}
$$

and the velocity gradient are relevant. Motion of the QS along curved paths round the poles within the horizontal plane is not relevant at all as long as no horizontal velocity gradient arises. Therefore, a particle resting within the velocity (gravitational) field of the spherically symmetric source outside the source at any point $(r, \theta, \phi)$ from the equator to the poles, will have an implicit velocity $\boldsymbol{V}_{i}(r)=-[\gamma M / r]^{1 / 2} \boldsymbol{e}_{\phi}$ and will be refracted downward at the same rate proportionally to the velocity gradient, that is, equal to the rotation rate of the local IR.

From the viewpoint of the local non-inertial laboratory observer, a gravitational pull is acting on objects within the laboratory. However, from the viewpoint of the local-IR this pull is exactly a centrifugal (fictitious) force toward the gravitational center that arises in response to the circular motion of the apparently resting laboratory within the local rotating IR under the real upward centripetal force exerted by the earthsurface. From the viewpoint of the non-inertial laboratory observer the wave fronts of the de Broglie matter waves propagating along $-\phi$ are dragged unevenly along $+\phi$ by the velocity field of the QS because the velocity in the velocity field increases for decreasing radial coordinate.

This uneven drag rotates the wave fronts at a rate equal to the angular velocity $\boldsymbol{W}(r)$ of the local IR about an axis overhead as shown in Figure 4, the rate of which increases with decreasing radial coordinate. In other words, the velocity field shifts unevenly the phase of the de Broglie waves, which leads to a refraction rate of the instantaneous $-\phi$ component of the propagation velocity vector $n$ at an angular velocity $\boldsymbol{W}(r)$. This generates an increasing vertical downward velocity component if the particle is free. This is the way the noninertial laboratory observer explains his observations. In reality the free particle simply goes on in its inertial path within each local rotating IR where it happens to be. The laboratory observer sees this apparent refraction rate because he together with the laboratory are implicitly moving along a circular path within the local rotating IR under the real upward centripetal force from the earth surface.

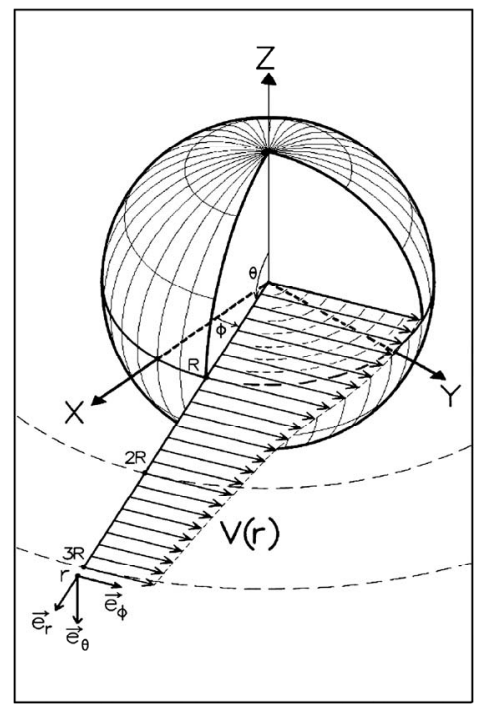

Figure 3. Variation of the velocity in the velocity field $\vec{V}(r)=[\gamma M / r]^{1 / 2} e_{\phi}$ of the QS along one radial line for a spherically symmetric source of radius $R$. $V(r)$ does not depend on the angular coordinates so that it is exactly the same along any radial direction from the equator to the poles.

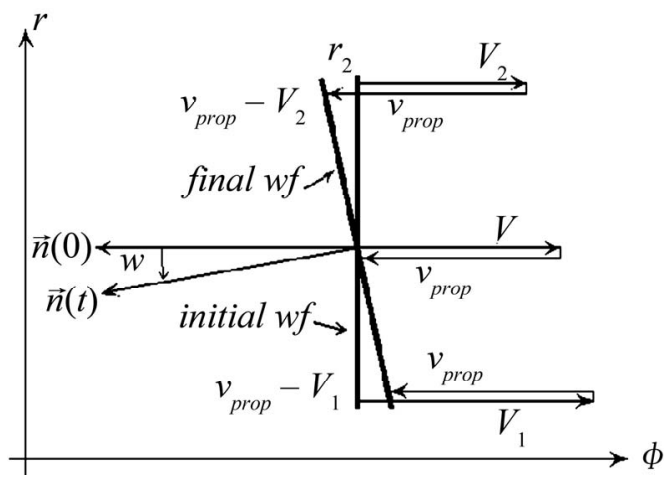

Figure 4. You are looking due north. The refraction rate $\vec{W}(r)$ of a wave front propagating toward $-\phi$, due to shear motion of the QS in the differential velocity field along $+\phi$. 
The above description of the gravitational physics is suitable as long as the velocity along the radial coordinate is sufficiently small. However, when the radial velocity component becomes relevant, its refraction must be considered too. This will be implemented in the next two sections.

\section{The Spacedynamic Gravitational Dynamics}

A particle that rests locally with respect to the QS at the equatorial plane of the Keplerian velocity field of the QS will be naturally moving along with the QS in a circular equatorial orbit. However, if this particle is given any velocity with respect to the QS within the equatorial plane, its orbit cannot be circular anymore, because this velocity with respect to the QS will be refracted. The only way to assure the possibility for the observed circular orbits of the planets is assuming that the circulation velocity of the QS, generating the gravitational field of the Sun, is consistent with the Keplerian motion of the planets:

$$
\begin{array}{cr}
\boldsymbol{V}(r)=(\gamma M / r)^{1 / 2} e_{\phi} \quad & (r>R) \\
\boldsymbol{V}(r)=V(R)\left[\left(3-r^{2} / R^{2}\right) / 2\right]^{1 / 2} e_{\phi} & (r<R)
\end{array}
$$

Equations (1a) and (1b) describe the velocity field of a homogeneous spherical mass respectively outside and inside a spherically symmetric and homogeneous mass distribution. In reality the planetary masses attenuate a little bit the velocity field of the Sun so that the decay of the velocity with distance falls a little bit lesser than given by Equation (1). This will say that the gravitational attraction by the solar system becomes a little bit stronger outside the solar system, which explains the Pioneer 10 and Pioneer 11 anomalies.

If the velocity of a planet with respect to the QS is zero then refraction of this zero velocity has no effects. On one hand, this is the only possibility of explaining the nearly circular orbital motions of the planets without the need of postulating fictitious gravitational forces or any other fictitious cause. On the other hand, all the experimental observations (please see Sections 9 and 10) consistently indicate that effectively the planets of the solar system closely rest with respect to the QS. It moreover will be found that this also is closely the situation of the stars within the galaxies throughout the universe (see Section 7).

A particle, resting with respect to the non-rotating coordinate axes (XYZ), will be moving along $-\phi$ with respect to the QS at the implicit velocity:

$$
\boldsymbol{v}_{\text {prop }}(r)=\boldsymbol{V}_{i}(r)=-(\gamma M / r)^{1 / 2} e_{\phi}
$$

where $\boldsymbol{V}_{i}(r)$ is the implicit propagation velocity. This velocity is implicit because it is the velocity of a particle with respect to the QS when it rests with respect to the (XYZ) axes and hence it cannot be specified with respect to the (XYZ) axes.

We still do not know the exact rates at which the velocity components of the propagation velocity $\boldsymbol{v}_{\text {prop }}$ of a particle with respect to the QS within the velocity field Equation (1) are refracted. These rates can be found precisely enough by meticulously calculating the orbital velocity $\boldsymbol{v}_{\text {orb }}$ of a particle in an elliptic equatorial orbit using Newtonian gravitation and considering that this orbital velocity is the vector composition of the propagation velocity $\boldsymbol{v}_{\text {prop }}$ and of the velocity of the QS given by Equation (1):

$$
\boldsymbol{v}_{\text {orb }}=\boldsymbol{V}(r)+\boldsymbol{v}_{\text {prop }}
$$

Figure $\mathbf{5}$ is a highly precise graphical representation that shows $\boldsymbol{v}_{\text {orb }}, \boldsymbol{v}_{\text {prop }}$ and $\boldsymbol{V}(r)$ for a large number of positions along an elliptic orbit with eccentricity $\varepsilon=0.5$. In this figure we can see how $\boldsymbol{v}_{\text {prop }}$ changes in magnitude and direction and precisely obtain the refraction rates of the $r, \phi$ and the $\theta$ velocity components of $\boldsymbol{v}_{\text {prop }}$. While the refraction rate of the $\phi$ component of $\boldsymbol{v}_{\text {prop }}$ can be read on top of the figure to be equal to the angular displacement in the orbit, that of the $r$ component can be seen at the left to be opposite and only one half the rate of the $\phi$ component. The $\theta$ component is not refracted at all because the velocity field has no velocity gradient in the $\theta-\phi$ plane. Equations (4) give the refraction rates of respectively the $r, \phi$ and the $\theta$ components of the propagation velocity as angular velocities:

$$
\begin{aligned}
& \boldsymbol{W}_{r}(r)=-\frac{1}{2}\left[\gamma M / r^{3}\right]^{1 / 2} \boldsymbol{e}_{\theta} \\
& \boldsymbol{W}_{\phi}(r)=\left[\gamma M / r^{3}\right]^{1 / 2} \boldsymbol{e}_{\theta} \\
& \boldsymbol{W}_{\theta}(r)=0
\end{aligned}
$$

The refraction rate of the $r$ component, expressed by Equation (4a) just compensates for the variations of the velocity field and therefore assures the free-fall from rest along strictly vertical line and the conservation of the angular momentum about the gravitational center. The behavior of sound waves propagating within whirl wind is similar [23-25]. Compression and stretching of the wavelength $(\lambda)$ of the de Broglie matter waves, due to the deformation of the QS in the velocity field Equation (1) too must be considered. Variations of the de Broglie wavelength affect the propagation velocity $\boldsymbol{v}_{\text {prop }}$ as given by de Broglie's equation $p=m v=h / \lambda$, where $h$ is Plank's constant. However, the refraction rates, expressed by Equation (4), have been evaluated for strictly the $r$ and $\phi$ velocity components along which there is no $\lambda$ contraction nor stretching and hence there is no need 
to explicitly include these effects. These effects however are incorporated in the effective angular velocities expressed by Equation (4).

In terms of Equation (4b), the instantaneous gravitational acceleration of particles initially resting within a laboratory fixed with respect to the (XYZ) axes and hence moving with respect to the QS with the implicit velocity Equation (2) can be calculated by the vector product to be:

$$
\boldsymbol{g}(r)=\boldsymbol{W}_{\phi}(r) \times \boldsymbol{V}_{i}(r)=-\gamma M / r^{2} e_{r}
$$

This is the instantaneous acceleration with respect to the (XYZ) coordinate axes toward the gravitational center from the viewpoint of the non-inertial laboratory observer. This gravitational acceleration field $\boldsymbol{g}(r)$ does not depend on the angular coordinates, it is spherically symmetric. From the viewpoint of the non-inertial laboratory observer, it is the rate at which vertical downward velocity is created due to the refraction rate of the local implicit velocity vector, which is the instantaneous gravitational acceleration. In reality this acceleration field is apparent (centrifugal) because it has been ob- tained from the viewpoint of the local non-inertial laboratory reference. From the viewpoint of the local rotating IR it is a centrifugal acceleration field toward the gravitational center. The judgment of the observer in the non-inertial earthbased laboratory reference comes from the fact that his reference is moving about an axis overhead within the local oppositely rotating IR about the same overhead axis. This axis can be found to lie overhead at a distance exactly equal to the distance to the gravitational center. Equation (5) does not contain the angular coordinates because the refraction rate $\boldsymbol{W}_{\phi}(r)$, given by Equation (4b) is proportional to the velocity gradient of the velocity field Equation (1) that has the same magnitude for all $\theta$ and $\phi$ and points to the gravitational center from the equator to the poles. Hence $\boldsymbol{g}(r)$ is a central spherically symmetric field of accelerations.

In order to calculate the free fall along large radial distances, it is necessary to take into account the refraction rate of the radial velocity component $v_{r}$ too. This refraction rate is opposite to that of the $\phi$ component (see Equation (4a)).

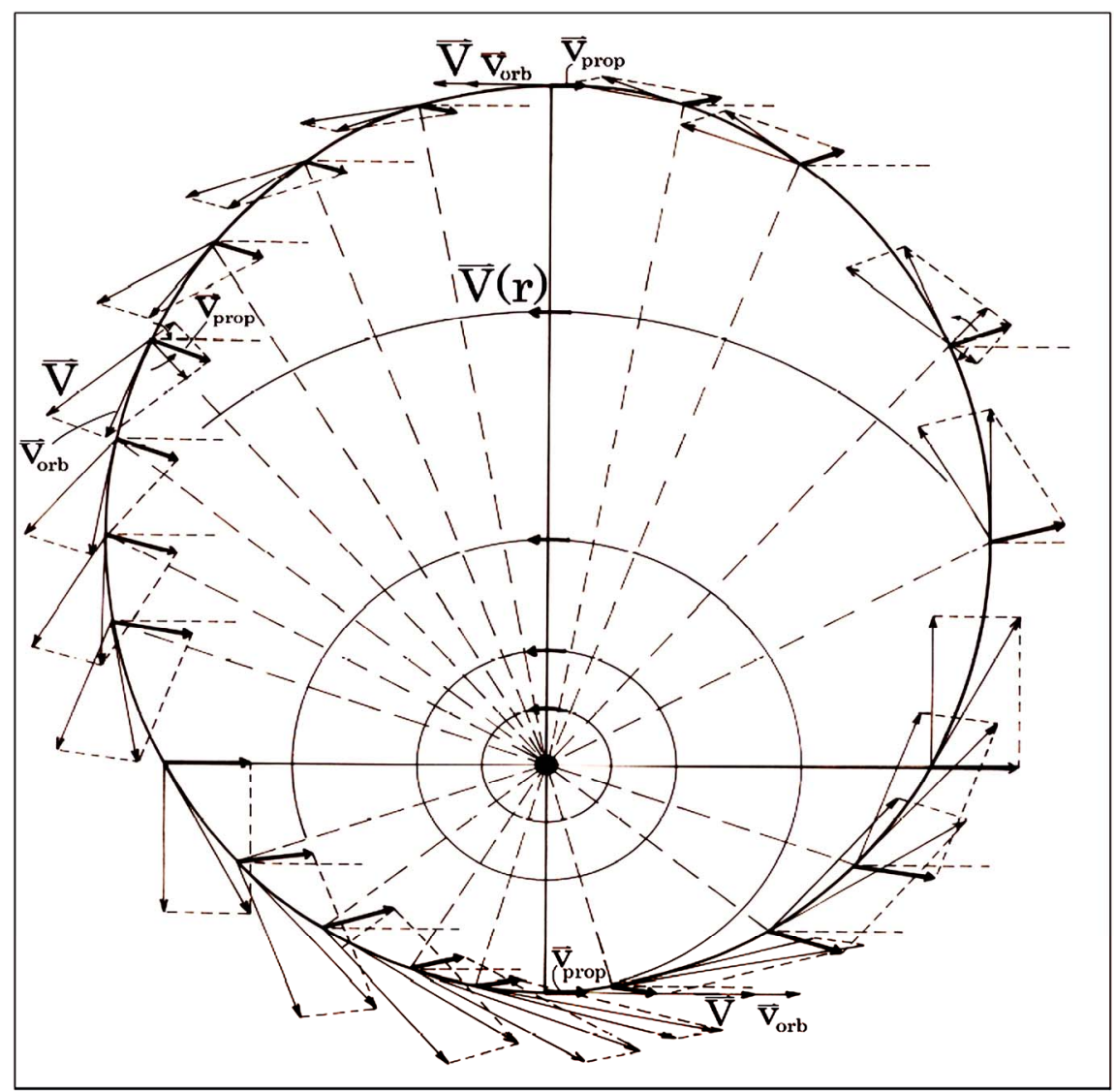

Figure 5. The figure is a very precise graphical representation. It displays $\vec{v}_{o r b}, \vec{v}_{p r o p}$ and $\vec{V}(r)$ for a large number of points along an elliptical orbit with eccentricity $\varepsilon=0.5$. The behavior of $\vec{v}_{p r o p}$ in magnitude and direction can precisely be read in the figure. 
The effects caused by the rotations in Equations (4) can be treated formally in terms of the elementary differential equation:

$$
\frac{\mathrm{d} v}{\mathrm{~d} t}=\mathrm{A} \boldsymbol{v}
$$

where $v$ is the column matrix of the $r$ and the $\phi$ velocity components:

$$
\boldsymbol{v}(t)=\left(\begin{array}{l}
v_{r}(t) \\
v_{\phi}(t)
\end{array}\right)
$$

and $A$ is the matrix:

$$
\mathrm{A}(r)=\left(\begin{array}{cc}
0 & W_{\phi}(r) \\
-W_{r}(r) & 0
\end{array}\right)=\left(\begin{array}{cc}
0 & W(r) \\
\frac{W(r)}{u} & 0
\end{array}\right)
$$

Here $W_{r}=-\frac{1}{2}\left[\gamma M / r^{3}\right]^{1 / 2}, \quad W_{\phi} \equiv W=\left[\gamma M / r^{3}\right]^{1 / 2}$ are given by Equation (4) and $u=2 M /(M+m) \approx 2$ for $m \ll M$ and is equal to 1 for $m=M$. This expression for $u$ is obtained from conservation of energy and linear momentum and accounts for the asymmetric distribution of kinetic energy between $m$ and $M$ as well as for the explicit time dependence of the velocity field $\boldsymbol{V}(r)$ due to motions of the source $M$ with respect to the center of mass under the field of $m$.

If $\boldsymbol{v}_{0}$ is the initial velocity, then the solution of Equation (6) is:

$$
\begin{aligned}
\boldsymbol{v}(t) & =\boldsymbol{v}_{0} \operatorname{Exp}\left[\int_{0}^{t} A\left(r\left(t^{\prime}\right)\right) \mathrm{d} t^{\prime}\right] \\
& =\sum_{n=0}^{\infty} \frac{1}{n !}\left(\begin{array}{cc}
0 & \Theta(t) \\
\frac{\Theta(t)}{u} & 0
\end{array}\right)\left(\begin{array}{l}
v_{r}(0) \\
v_{\phi}(0)
\end{array}\right) \\
& =\left(\begin{array}{cc}
\cosh \left(\frac{\Theta(t)}{\sqrt{u}}\right) & \sqrt{u} \sinh \left(\frac{\Theta(t)}{\sqrt{u}}\right) \\
\frac{1}{\sqrt{u}} \sinh \left(\frac{\Theta(t)}{\sqrt{u}}\right) & \cosh \left(\frac{\Theta(t)}{\sqrt{u}}\right)
\end{array}\right) \times\left(\begin{array}{c}
v_{r}(0) \\
v_{\phi}(0)
\end{array}\right)
\end{aligned}
$$

where $\Theta$ is the integrated angular displacement:

$$
\begin{aligned}
\Theta(t) & =\int_{0}^{t} W\left[r\left(t^{\prime}\right)\right] \mathrm{d} t^{\prime} \\
& =\int_{r_{0}^{C M}}^{r^{C M}}\left[\frac{\gamma M}{\left(r^{C M}+R^{C M}\right)^{3}}\right]^{1 / 2} \frac{\mathrm{d} r^{C M}}{\dot{r}^{C M}} \\
& =-\sqrt{u} \cosh ^{-1}\left[\frac{r_{0}^{C M}}{r^{C M}}\right]^{1 / 2}
\end{aligned}
$$

Here use has been made of $\dot{r}^{C M}=\mathrm{d} r^{C M} / \mathrm{d} t^{\prime}$. The superscripts $(C M)$ define quantities taken with respect to the center of mass. An expression for $\dot{r}^{C M}$ can be found equating the total kinetic energy of the system in the $C M$ reference to the total variation of the potential energy and using the definition of the factor $u$ to relate coordinates and velocities of $m$ and $M$. Inversion of Equation (10) leads to:

$$
\begin{aligned}
\cosh \frac{\Theta(t)}{\sqrt{u}} & =\left[r_{0}^{C M} / r^{C M}\right]^{1 / 2}=\left[r_{0} / r(t)\right]^{1 / 2} \\
\sinh \frac{\Theta(t)}{\sqrt{u}} & =\left[\left(r_{0}^{C M}-r^{C M}\right) / r^{C M}\right]^{1 / 2} \\
& =\left[\left(r_{0}-r(t)\right) / r(t)\right]^{1 / 2}
\end{aligned}
$$

where the last equality is easily proved. With this result Equation (9) becomes:

$$
\begin{aligned}
\left(\begin{array}{l}
v_{r}^{C M}(t) \\
v_{\phi}^{C M}(t)
\end{array}\right)= & \left(\begin{array}{cc}
\sqrt{r_{0} / r} & \sqrt{u\left(r_{0}-r\right) / r} \\
\sqrt{\left(r_{0}-r\right) / u r} & \sqrt{r_{0} / r}
\end{array}\right) \\
& \times\left(\begin{array}{l}
v_{r}^{C M}(t=0) \\
v_{\phi}^{C M}(t=0)
\end{array}\right)
\end{aligned}
$$

For free fall on from $r_{o}$ and initial rest $\left[v_{r}(0)=0\right.$ and $\left.v_{\phi}(0)=V_{i}\left(r_{0}\right)=-(\gamma M / r)^{1 / 2} e_{\phi}\right]$, the solution of Equation (6) is:

$$
\begin{aligned}
& v_{r}(t)=\left[\frac{2 M}{M+m}\left(\frac{\gamma M}{r(t)}-\frac{\gamma M}{r_{0}}\right)\right]^{1 / 2} \\
& v_{\phi}(t)=V_{i}\left(r_{0}\right)\left[\frac{r_{0}}{r}\right]^{1 / 2}=\left[\frac{\gamma M}{r(t)}\right]^{1 / 2}
\end{aligned}
$$

Equation (13a) is just the well known expression for the observed vertical free fall on from rest at $r_{o}$ and Equation (13b) is just the implicit velocity as a function of the radial position $r$, which shows that the refraction of the radial velocity component just compensates for the increase of the velocity field with the decrease of the radial coordinate. This assures that the particle falls on from rest along a perfectly vertical (radial) path and also assures conservation of the angular momentum about the gravitational center in motions within gravitational fields.

From the viewpoint of spacedynamics, the gravitational potential is really a centrifugal potential integrated from infinity to a radial position $r$. The angular velocity of a mass $m$ resting at $(r, \theta, \phi)$ round an overhead axis within the gravitational field of a mass $M(m \ll M)$ is:

$$
\boldsymbol{W}(r)=-\left[\frac{\gamma M}{r^{3}}\right]^{1 / 2} \boldsymbol{e}_{\theta}
$$

Integration of the real upward centripetal force per unit mass $\left(W^{2} r\right)$ for motion from $r=\infty$ to $r$ gives:

$$
\chi(r)=-\int_{\infty}^{r} W^{2} r^{\prime} \mathrm{d} r^{\prime}=-\gamma M \int_{\infty}^{r}\left[r^{\prime-2} \mathrm{~d} r^{\prime}\right]=-\frac{\gamma M}{r}(
$$


This variation of the centripetal potential depends only on the radial coordinate and hence is a spherically symmetric scalar potential field. It essentially measures the mechanical work realized per unit mass along a uniform motion from $r^{\prime}=\infty$ to $r^{\prime}=r$.

\section{Symmetry of $g(r)$ with Orbital Motions}

Obviously, from the viewpoint of spacedynamics, the orbital motion of the planets round the Sun can certainly not be looked anymore as motion within a usual inertial reference valid in the whole region because, within the gravitational field, each local IR is valid rigorously only within an infinitesimal region of space. However, on examining the orbital motion of bodies within the velocity field of the QS Equation (1), it will be found that the spacedynamic gravitational mechanism is perfectly symmetric with direct and retrograde orbital motions and that the orbital motion of bodies round a gravitational source works exactly as motions within an extended inertial reference under a central field of fictitious gravitational forces as conceived in Newtonian gravitation.

Consider free-fall experiments, performed within a laboratory on the surface of a planet that rotates about the $\mathrm{Z}$ axis (the axis of the velocity field) at an angular velocity $\omega$. A body within the laboratory will be moving with respect to the QS at a velocity of propagation:

$$
\begin{aligned}
v_{\text {prop }}(\theta) & =\boldsymbol{V}_{i}(R)+v_{\text {rot }}(\theta) \\
& =-\left[(\gamma M / R)^{1 / 2} \mp \omega R \sin \theta\right] e_{\phi}
\end{aligned}
$$

where $\boldsymbol{V}_{i}$ is the implicit velocity Equation (2) on the planet's surface, $v_{r o t}(\theta)$ is the local ordinary velocity of the laboratory along the $\phi$ coordinate due to the planet's rotation, which depends on the latitude via $\sin \theta, R$ is the radius of the rotating planet and the upper and lower signs are respectively for direct and retrograde rotation of the planet with respect to that of the velocity field of the QS.

However, the orbital motion along the $\phi$ coordinate also affects the direction of $v_{\text {prop }}$ with respect to the gravitational center by a usual trigonometric rotation rate as can be seen from Figure 6. Hence, the effective angular velocity is:

$$
\begin{aligned}
\omega_{\text {eff }}(\theta) & =\left[V / R \pm\left(v_{r o t}\right) / R\right] e_{\theta} \\
& =\left[\left(\gamma M / R^{3}\right)^{1 / 2} \pm \omega \sin \theta\right] e_{\theta}
\end{aligned}
$$

where the same convention for the upper and the lower signs as in Equation (16) is used.

Considering the effective propagation velocity and the effective angular velocity, the effective gravitational acceleration is:

$$
\boldsymbol{g}_{\text {eff }}(\theta)=\boldsymbol{\omega}_{\text {eff }} \times \boldsymbol{v}_{\text {prop }}=-\left[\gamma M / R^{2}-\omega^{2} R \sin ^{2} \theta\right] e_{r}
$$

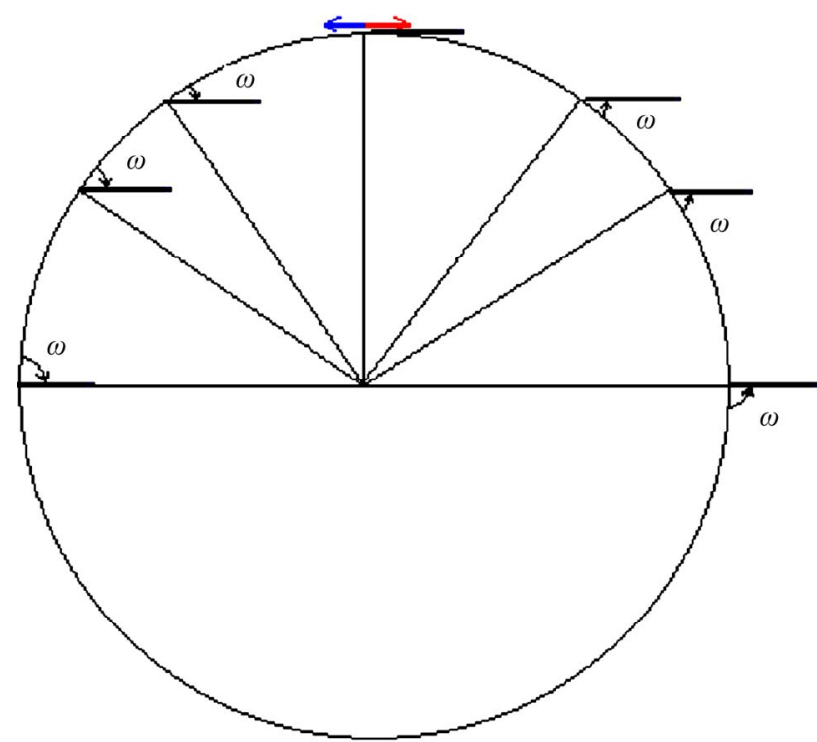

Figure 6. You are looking due north. Due to the orbital motion $\vec{v}_{o r b}$, the direction of $\vec{v}_{p r o p}$ with respect to the gravitational center changes at a rate equal to the angular velocity in the orbit.

Equation (18) shows that $\boldsymbol{g}_{\text {eff }}(\theta)$ is perfectly symmetric for direct or retrograde rotation (orbital motion of body) of the planet. The first term in the right hand side describes acceleration toward the gravitational center [see Equation (5)], while the second term describes an outward centrifugal acceleration. However, this is only the viewpoint of the local non-inertial laboratory observer. From the viewpoint of the local-IR, the first term is a centrifugal effect that arises because of the circular motion of the apparently resting earthbased laboratory within the local rotating IR. The second term seems to be an outward centrifugal term. It however describes simply the decrease of the first term due to the velocity of the body and the earthbased laboratory along the $\phi$ coordinate, as consequence of the planet's rotation. This result shows that the spacedynamic gravitational mechanism is symmetric for direct or retrograde equatorial orbital motions, as effectively observed.

For strictly circular polar orbits of radius $r, \boldsymbol{v}_{\text {prop }}$ has a component $v_{\phi}$ along $\phi$

$$
v_{\phi}=\vec{V}_{i}(r)=-[\gamma M / r]^{1 / 2} e_{\phi}
$$

as well as a component $v_{\theta}$ along

$$
v_{\theta}= \pm[\gamma M / r]^{1 / 2} e_{\theta}
$$

While $v_{\phi}$ generates the gravitational acceleration field $\boldsymbol{g}(r)=-\gamma M / r^{2} e_{r}$, see Equation (5), the $\theta$ component is not affected directly by spacedynamics because the velocity field Equation has no velocity gradient in the $(\theta, \phi)$ plane. Hence, the effective gravitational acceleration is: 


$$
\boldsymbol{g}_{\text {eff }}(r)=-\left[\gamma M / r^{2}-\left(v_{\theta}^{2}\right) / r\right] e_{r}
$$

Depending on the magnitude of the $v_{\theta}$ velocity component, the effect of the $v_{\phi}$ component enforces the particle into a parabolic, circular or elliptic path. The curved motion along the $\theta$ coordinate gives rise to a genuine centrifugal effect. More general motions combine the effects expressed by Equations (18) and (21). Although an analytical solution of Equation (6) for such general motions is not at all simple, numerical methods may be quite effective. Figure 5 displays in reality a number of snapshots of such a solution for an elliptical equatorial orbit.

The results expressed by Equations (5), (13), (18) and (21) are indeed remarkable. Although the local-IR changes from point to point within the velocity field Equation (1), the spherical symmetry of the velocity field ends up by creating a central spherically symmetric field of centrifugal accelerations toward the gravitational center. In the view of the observer in the non-inertial earthbased laboratory, this inward centrifugal acceleration field seems to be a gravitational acceleration that manifests it as a gravitational pull on the objects, simulating a central field of Newtonian gravitational forces. On the other hand, the ordinary motions of bodies with respect to the (XYZ) axes simulate outward centrifugal forces likewise circular motions within an inertial reference exactly as conceived in Newtonian mechanics. This explains why Newtonian gravitation works so well.

However, what is really astonishing and wonderful in this spacedynamic gravitational mechanism is the fact that within the velocity field of the real space Equation (1), in which any inertial reference can be valid at only one geometrical point, ends up in such a simple solution in which the orbital motions of the planets can be described as usual motions in a well defined (extended) inertial reference under a central force field. From the classical mechanics viewpoint, these orbital motions with respect to the (XYZ) coordinate axes can be seen as defined by the equilibrium of the central field of gravitational forces and the outward centrifugal forces due to the ordinary motions exactly as conceived in Newtonian mechanics. This shows that the effects of spacedynamics and of the ordinary motions are disconnected, which means that the implicit dynamics created by the velocity field Equation (1) and the ordinary dynamics due to the ordinary motions are orthogonal. Effectively, while the implicit velocity Equation (2) and the rotation rate of the earthbased laboratories about axes overhead have no meaning from the viewpoint of the (XYZ) coordinate axes, the ordinary motions and the rotations expressed by Equations (4) have no meaning from the viewpoint of the local IRs. This means that the effects of spacedynamics and the effects of the ordinary dynamics manifest them independently and effectively behave as orthogonal. If we choose to describe this gravitational dynamics from the viewpoint of the (XYZ) coordinate axes (the viewpoint of the non-inertial earthbased laboratory observer), we can mathematically account for this by expressing the ordinary motions of bodies as purely real quantities, while the implicit velocity Equation (2) and the rotation rate of the earthbased laboratories that are opposite to Equations (4) as purely imaginary quantities.

\section{Dark Matter}

It is well known since decades that the orbital velocity of the stars round the galactic nucleus falls not off with distance as predicted by the current theories of space and gravitation. Many published papers [32-34] display observed velocity profiles for the orbital velocity of the stars in galaxies as a function of distance from the galactic center. Figure 7 displays an observed velocity profile of our Milky Way galaxy together with the Keplerian rotation predicted by the current theories. From the viewpoint of these theories, the rotation rate of galaxies is much too fast to be accounted for by the gravitational force generated by their content of visible matter.

This galactic rotation rate has enforced the hypothesis that a huge amount of an exotic dark matter that acts gravity but does not scatter, absorb or emit electromagnetic radiation. Challengingly however, the amount of dark matter, necessary to account for the observed galactic rotation rate, must be about 5 times larger than that of the whole visible matter. To present date nobody has idea where such a huge amount of dark matter could be hided and what its nature is. Some authors even propose a distribution of dark matter that may explain the observed galactic rotation. Others however claim that whatever the distribution of dark matter, it cannot reproduce the observed galactic gravitational dynamics. The galactic rotation rate is actually one of the most serious impasses in the current theories of space and gravitation.

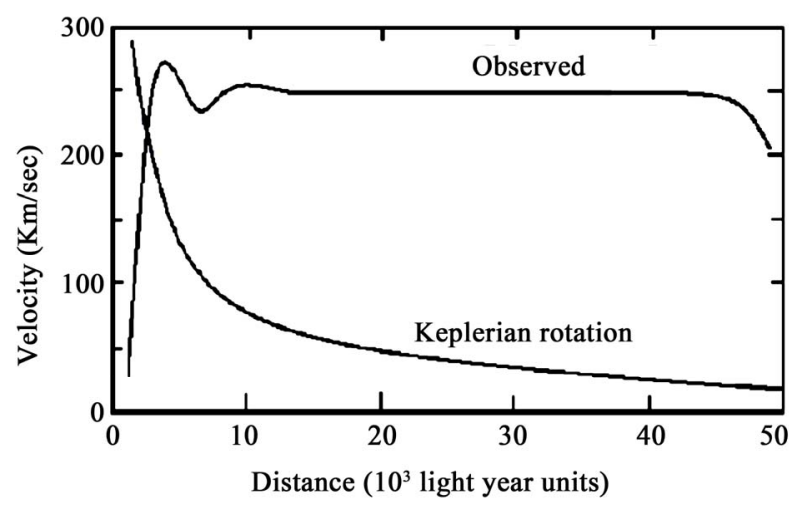

Figure 7. Profiles of the observed rotation rate of the Milky Way galaxy as a function of distance from the galactic nucleus and the Keplerian rotation rate. 
In the view of spacedynamics, the gravitational dynamics of the galaxies cannot be ruled by a Keplerian velocity field of the QS given by Equation (1) because their distribution of mass is not spherically symmetric. Galaxies have a central bulge of orbiting gases and stars that normally harbors a supermassive black hole. Beyond the bulge, up from about $10^{4}$ light years, they are formed by a swarm of stars in the form of a thin disk that extends out to about $5 \times 10^{4}$ light years. The stars are separated from each other by several light years so that most of the space within a galaxy is empty. Almost the whole mass of a galaxy is in the form of stars that are orbiting round the galactic nucleus under the collective self-consistent velocity (gravitational) field created by them. The density of the galactic matter undergoes some variation with distance due to their spiraled structure. Our Milky Way galaxy is an old barred spiral galaxy having a central bulge of about $10^{4}$ light years that is surrounded by a thin disk-shaped swarm of orbiting stars extending out to about $4.5 \times 10^{4}$ light years. The bulge contains a central region of lower mass density having a diameter of several thousand light years wherein it exhibits a bar shaped halo of stars and hot and dense flowing gases and within the bar it harbors a supermassive black hole.

What is especially relevant and from the viewpoint of spacedynamics in no way can be neglected in the galactic gravitational dynamics is the fact that almost the whole matter that creates the galactic velocity (gravitational) field of the QS is moving in nearly circular orbits round the galactic nucleus. Obviously each orbiting star carries with it its velocity (gravitational) field given by Equation (1). The stellar velocity fields of the QS are fairly well polarized and rotate all in the same sense, which determines the form of the galactic velocity field that rules the galactic gravitational dynamics.

In order to highlight the relevance of the effect of motion of the gravitational sources on the collective velocity field of the QS of a system of bodies gravitating in their self-consistent field, let us begin with the simple case of a binary system of two equal stars of mass $\mathrm{M}$ moving in the same circular orbit round the center of mass in the equatorial plane of the collective velocity field as shown in Figure 8. The dynamics of such a system from the viewpoint of the non-rotating $\mathrm{XY}$ coordinate system is fairly well described by Newtonian mechanics. Balance of the mutual Newtonian gravitational forces $\gamma M^{2} /\left(2 x_{o}\right)^{2}$ and of the centripetal forces $M v_{o}^{2} / x_{o}$ on each star, due to the orbital motion, define the orbital velocity $v_{o}$ round the center of mass $(\mathrm{CM})$ :

$$
v_{o}(r)=\frac{1}{\sqrt{2}}\left(\gamma M / 2 x_{o}\right)^{1 / 2}
$$

On the other hand, the velocity of a small test particle, orbiting round an equal isolated (static) mass $M$ at the same distance $2 x_{o}$ in a circular equatorial orbit, is considerably larger:

$$
v_{o}^{\prime}(r)=\left(\gamma M / 2 x_{o}\right)^{1 / 2}=\sqrt{2} v_{o}
$$

Obviously, from the viewpoint of the current gravitational theories there is nothing wrong with Equation (22) and Equation (23). However, from the viewpoint of spacedynamics, Equations (22) and (23) unveil a key feature that discloses the effect of motion of the gravitational source on its velocity field of the QS. This is an essential ingredient that rules the gravitational dynamics of a system of gravitating bodies in their collective velocity (gravitational) field. In Section IV it has been shown that the most obvious way to a particle to be moving in a circular orbit is commoving with the QS in the velocity field creating the gravitational field of a source $M$. Hence, Equation (23) describes the respective velocity field of the QS. Otherwise, the orbit would not be circular. However, this is certainly true as well in the case of the much lower orbital velocity of the binary system. Equation (22) too is the velocity of the QS at the position of $M_{1}$ and $M_{2}$ in the combined velocity field of the binary system. Hence, both Equations (22) and (23) describe the respective velocity fields of the QS. However, why are these velocities so different? The only possible reason for the reduced velocity of the QS of $M_{1}$ at the position of $M_{2}$ and that of $M_{2}$ at the position of $M_{1}$ in the binary system (Figure 8) is the orbital motion of respectively $M_{1}$ and $M_{2}$ round the center of mass. The orbital velocity of the binary system reduces the velocity field $V_{\text {lint }}$ of $M_{1}$ at the right-hand side of $M_{1}$ and also reduces $V_{2 i n t}$ of $M_{2}$ toward the left-hand side of $M_{2}$, as given by Equation (22) and as depicted in Figure 8. In the case of Equation (23) the CM of the system practically coincides with $M$, and therefore the velocity

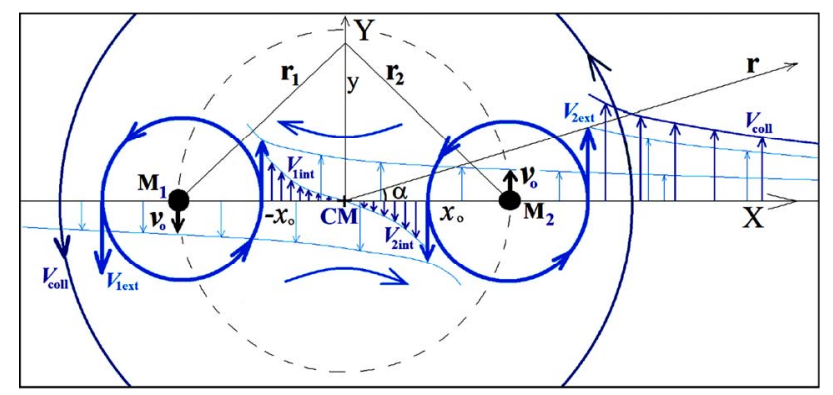

Figure 8. The orbital motion of a binary system of two masses $M_{1}=M_{2}=M$ round the center of mass $(\mathrm{CM})$ within the equatorial plane of the combined velocity field. Note that, while the velocity fields of the QS round each individual mass of the binary are symmetric from the viewpoint of the non-rotating references attached to each moving mass, from the viewpoint of the $\mathrm{CM}$ non-rotating $[\mathrm{X}, \mathrm{Y}]$ coordinate system with origin fixed to the $C M$, the velocity fields of $M_{1}$ and $M_{2}$ are larger outside than inside the binary orbit due to the orbital motion of the sources round the CM. 
field of the QS, due to it, is not affected at all and its velocity field is that of a static source exactly as given by Equation (1).

Consider now in addition a small test particle moving in the collective velocity field round the binary system within the orbital plane of the binary, however sufficiently far away in order to minimize the asymmetries of the binary field. The orbital velocity $v$ of such a test particle is of course:

$$
v(r)=(2 \gamma M / r)^{1 / 2}
$$

This expression for large $r$ too represents the velocity of the QS in the collective velocity field $V_{\text {coll }}$ round the binary system:

$$
V_{\text {coll }}(r)=(2 \gamma M / r)^{1 / 2}
$$

In order to reconcile the addition of the velocity fields of $M_{1}$ and $M_{2}$ outside the binary with the collective velocity field $V_{\text {coll }}(r)$ given by Equation (25), the same orbital velocity that reduces the velocity fields toward the inner side must enhance the velocity fields outward the binary. It must increase $V_{1}$ toward the left-hand side of $M_{1}$ and $V_{2}$ toward the right-hand side of $M_{2}$. At distances $r$ much larger than $2 x_{o}$, the addition of the velocity fields of $M_{1}$ and $M_{2}$ must reproduce the value given by Equation (25). Note that addition of velocity fields must obey the sum rule for velocity fields:

$$
V=\left(V_{1}^{2}+V_{2}^{2}\right)^{1 / 2}
$$

This rule arises because in the expression for the velocity field Equation (1) only the square root of the source mass is effective. Accordingly, addition of the velocity fields as a function of $x$ along the $\mathrm{X}$ axis outside of the binary in Figure 8 conforms to the equation:

$$
\frac{2 \gamma M}{x}=\frac{1}{2} \frac{\gamma M}{x_{o}+x}+V_{1,2}^{2}
$$

Solving for $V_{\text {ext }}=V_{1,2}$, along the $\pm X$ axis for large $x$, which means large $r$, the velocity fields of $M_{1}$ as well as of $M_{2}$ outside the binary are given by:

$$
V_{e x t}=\sqrt{\frac{3}{2}} \sqrt{\frac{\gamma M}{r}}
$$

This shows that effectively the same orbital velocity that reduces the velocity field of $M_{1}$ (Equation (22)) by a factor $(1 / 2)^{1 / 2}$ toward the right-hand side of $M_{1}$ in Figure 8 and the velocity field of $M_{2}$ toward the left-hand side of $M_{2}$, enhances the velocity field of $M_{1}$ and $M_{2}$ by a factor $(3 / 2)^{1 / 2}$ toward the right-hand side of $M_{2}$ and that of $M_{1}$ toward the left-hand side of $M_{1}$. This is of course not for nothing. The reason for this clearly is the orbital motion of the sources. However, from the viewpoint of spacedynamics, the individual sources are resting with respect to the QS in the collective velocity field, thereby preserving the spherical symmetry of their velocity fields.

According to Equation (26), taking into account Equations (22) and (28), the collective velocity field of the QS outside and round the binary as a function of $x$ is given by:

$$
V_{e x t}=\sqrt{\frac{3}{2} \frac{\gamma M}{x-x_{o}}+\frac{1}{2} \frac{\gamma M}{x_{o}+x}}
$$

For large $x$, which means large $\mathrm{r}$, this equation tends to equal Equation (25).

In-between the orbiting bodies of the binary system the velocity fields of $M_{1}$ and $M_{2}$, are reduced by their orbital velocity and are opposite to each other and opposite to the external velocity field. Close to $M_{1}$ the velocity field of $M_{1}$ dominates and close to $M_{2}$ the velocity field of $M_{2}$ is dominant. The resultant collective velocity field in-between the two masses as a function of $x\left[V_{\text {int }}(x)\right]$ along the $\mathrm{X}$ axis is given by:

$$
\begin{aligned}
V_{\text {int }}(x) & =\sqrt{\left|V_{1}^{2}-V_{2}^{2}\right|} \\
& =\sqrt{\left|\frac{1}{2} \frac{\gamma M_{1}}{x_{o}+x}-\frac{1}{2} \frac{\gamma M_{2}}{x_{o}-x}\right|}
\end{aligned}
$$

For $x=0 \quad V_{\text {int }}(x)=0$, while for $x=x_{o} \quad V_{\text {int }}(x)$ points downwards in Figure 8 and is large near $M_{2}$, for $x=-x_{o} \quad V_{\text {int }}(x)$ points upwards and is large near $M_{1}$. For other directions, $V_{\text {int }}$ must be found by adequate vector composition in conformity with Equation (26).

Along the axis of $\mathrm{Y}$ the collective velocity field may be expressed by:

$$
V(y)=\sqrt{\frac{2 \gamma M y^{2}}{\left(x_{o}^{2}+y^{2}\right)^{3 / 2}}}
$$

However, this equation is certainly not good close to the binary because there the collective velocity field is considerably affected by the flow continuity and conservation of volume. However, for large $y$ Equation (31) too approximates Equation (25). Note that the form of the velocity fields as expressed by Equations (30) and (31) remain invariant with time only if the $X Y$ coordinate axes rotate with the binary. Note also that within the binary orbit the rotation of the velocity field expressed by Equations (30) round the CM and as shown in Figures 8 and 9 is opposite to the orbital motion of the binary as well as opposite to the external velocity field and moreover opposes the effective velocity field as a function of $y$ along the $\mathrm{Y}$ axis. It is clear that within the orbit of the binary the velocity field is strongly reduced and that a stagnation point exists at the CM where the velocity of the QS falls to zero. The formation of such a region of depressed 
velocity becomes even more evident if on the same circular orbit there are four or more equally spaced bodies of mass $M$ orbiting in the same sense round the center of mass under their self-consistent velocity (gravitational) field as shown in Figure 9.

With the increase of the number of masses moving in the same orbit, the opposite velocity field of the QS expressed analogously as in Equation (30) will become more and more dominant in the whole interior of the orbit, while outside the loop the direct collective velocity field will be enhanced by the orbital velocity of more and more sources. In order to advance closer toward the realistic situation of a galactic system, consider now multiple concentric orbiting loops with larger and larger radii orbiting in the same sense and each loop containing a large number of bodies. It is not difficult to understand that the external loops act in the sense of reducing the velocity field of the QS created by the inner loops while enhancing the velocity field outward. The reason of all this obviously is the orbital motion of the gravitational sources in the successive loops and acts in the sense of counteracting the decrease of the collective velocity field with distance from the center dictated by the Keplerian rotation. If the mass density as a function of distance from the center of mass is constant, the velocity field is expected to increase with distance and, depending on the variation of the mass density and the polarization of the velocity fields, a central region with a retrograde rotation may form. In some galaxies such retrograde rotation is effectively observed. NGC 7331 is an example of a galaxy which has a bulge that is rotating in the opposite sense to that of the rest of the disk [35]. However, other well known examples exist. Moderate decrease of the mass density with distance may lead to nearly constant velocity as a function of distance as observed in our Milky Way galaxy. Only beyond the border of such disk shaped rotating systems can the velocity field decrease freely according to a Keplerian rotation.

The above analysis evidences that spacedynamics predicts qualitatively the observed galactic rotation rate and provides the tools to settle the galactic gravitational dynamics. It may be interesting to note that within the solar system the planets too are expected to induce a very weak but non-vanishing attenuation in the $(1 / r)^{1 / 2}$ dependence of the Keplerian velocity (gravitational) field. Hence, the radial dependence of the velocity field becomes truly Keplerian only beyond the border of the solar system. This will say that the solar gravitational acceleration increases a little bit beyond the border of the solar system. This may explain the Pioneer anomaly, which is a very small but consistent increase in the gravitational attraction of the Pioneer 10 and Pioneer 11 spacecrafts by the Sun, observed beyond the border of the solar system [36,37]. The local perturbation of the

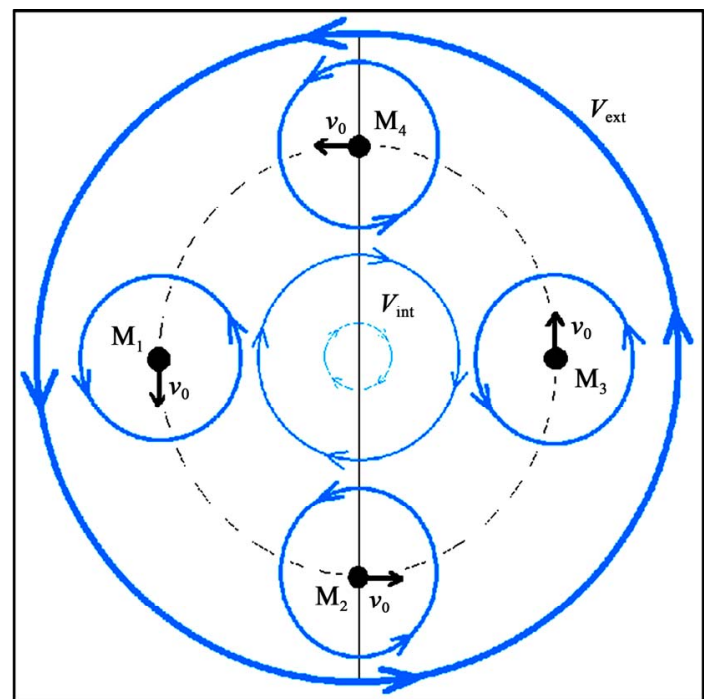

Figure 9. The figure sketches the collective velocity field generated by four equal masses moving in the same circular orbit round the center of mass. Note that within the orbit the velocity field of the QS circulates in the opposite sense with respect to the external velocity field and that by expressions analogous to Equations (29) and (30) $V_{i n t}$ is much smaller than $V_{\text {ext }}$.

solar velocity field by a planet may also cause another gravitational anomaly that is responsible for the observed anomalous acceleration of spacecrafts during close to Earth flybys [38].

Within the disk of the Milky Way galaxy the stars are moving round the galactic center in orbits that are closely circular. From the viewpoint of spacedynamics, this is evidence that these stars are commoving with the QS in the galactic velocity field of the QS. Moreover, the null results of the Michelson experiments that searched for light anisotropy due to the orbital and cosmic motion of Earth demonstrate that, despite the motion of the solar system at about $250 \mathrm{~km} / \mathrm{sec}$ round the galactic center, its velocity with respect to the QS is closely zero. Consonantly the velocity of all the stars of the galaxy within the galactic disk may be closely zero too. This will say that the equator of the velocity field of the QS creating the galactic gravitational field coincides with the galactic disk and that the motion of stars along the circular orbits round the galactic center are in fact commoving with the QS in the galactic velocity field. Hence, the function that describes the observed circular orbital velocities of the stars within the galactic disk also describes well the galactic velocity field of the QS. This will say that the velocity of light is expected to be isotropic with respect to the stars and that clocks locally commoving with the stars go not slow and run all at closely the same rate. These circular orbital motions of the stars are of course not constrained by gravitational forces. According to spacedynamics, the stars are essentially carried around 
by the moving QS in the galactic velocity field of the QS, analogously as the planets are carried around the Sun by the solar velocity field of the QS that creates the solar gravitational field. This corresponds locally to physical rest of the stars with respect to the moving QS so that these orbital motions need not to be explained anymore, because it is space itself that so moves.

Another important parameter in the gravitational dynamics is the gravitational potential $[U(r)]$. The gravitational potential has a very simple relation with the (circular) orbital velocity $V_{\text {orb }}(r)$ :

$$
U(r)=-V_{o r b}^{2}(r)
$$

This relation that is dictated by the Virial Theorem is quite general. Hence, knowing the orbital velocity, the gravitational potential can be directly calculated and vice-versa. Several authors have computed the gravitational potential $[U(r)]$ as a function of the distance from the galactic nucleus for our Milky Way galaxy as well as for other galaxies with base in the conventional theories of gravitation and taking into account the visible star density as a function of distance (see for instance reference [39]). Systematically these gravitational potentials are all inconsistent with the observed orbital motions of the stars.

Figure 10 displays (red) the usual gravitational potential $U(r)=-\gamma M / r$, calculated by assuming that the $1 / r$ dependence is related to the distance from the galactic center and using as a base the value of $U$ calculated from Equation (32) near the galactic border. This $1 / r$ dependence is totally inconsistent with observations within the galactic disk. Figure $\mathbf{1 0}$ in addition displays (blue) the gravitational potential, as calculated by Equation (32) using the observed orbital velocities of the stars round the galactic center given in Figure 7 and assuming that this velocity profile beyond the galactic border has the form of the Keplerian rotation. In this assumption the effect of the large number of globular star clusters that move beyond the galactic border analogously as planets move in the solar system and may induce some attenuation in the $(1 / r)^{1 / 2}$ dependence of the velocity field, has not been considered. The gravitational potential, determined with base in Equation (32), is considerably more leveled within the galactic disk than the usually computed potentials. This is what is qualitatively predicted by spacedynamics. In the view of spacedynamics, the velocity gradient within this region is very low so that the gravitational acceleration of a non-orbiting body, located within the galactic disk, toward the galactic nucleus is very weak. This will say that moving a body along the galactic radius within the galactic disk costs relatively little work. However, on from the galactic border, the velocity field of the QS is expected to fall steeply and to retake the $(1 / r)^{1 / 2}$ dependence of the

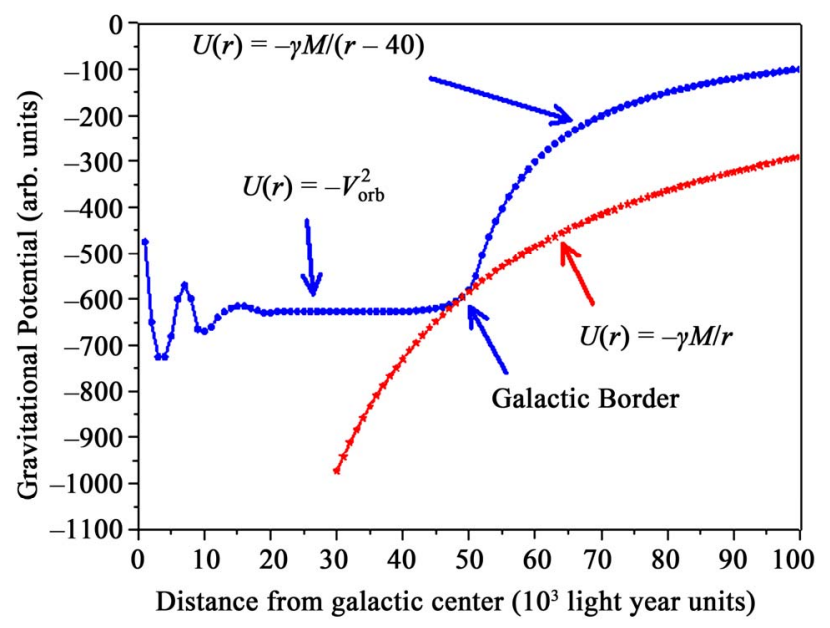

Figure 10. The galactic gravitational potential as a function of distance from the galactic center, showing the gravitational potential $[U(r)]$; (red) With the usual $1 / r$ dependence, calculated with base in the value of $U$ at the galactic border and taking $r$ as the distance to the galactic center; (blue) The gravitational potential as given by Equation (32) up to the galactic border and assuming that beyond the galactic border the velocity follows the Keplerian rotation, however calculated in terms of the distance $r$ on from the galactic border.

Keplerian rotation. This Keplerian rotation may not be related to the distance from the galactic center but to the distance on from the galactic border. Correspondingly, in this region the velocity gradient toward the galactic center is very large and hence beyond the galactic border the gravitational potential too falls off steeply as the inverse of the distance on from the galactic border. This will say that near the galactic border the gravitational acceleration toward the galactic center is very strong.

In the view of spacedynamics, the stars need not to be acted on by forces do move along their circular orbits round the galactic center, but are simply carried around by the moving space in the galactic velocity field. Although an exact determination of the galactic velocity field with base in the spacedynamic gravitational physics has not been worked out, the above analysis gives convincing evidence that spacedynamics predicts qualitatively even with details the observed velocity profiles of the galactic rotation rate and the galactic gravitational dynamics without the need of dark matter. Even the possibility of the unbelievable retrograde rotation of the inner part of a galaxy, observed in some galaxies, is a natural outcome of spacedynamics.

\section{Dark Energy}

The discovery of the accelerated expansion of the universe $[40,41]$ has add new features and new challenges to our understanding. To now this accelerated expansion has no physical explanation. It is usually imputed to a 
new kind of (positive) potential energy, simply named dark energy. Explanation of the accelerated expansion of the universe has also been attempted in terms of the cosmological term in Einstein's equation for the spacetime curvature. This however is not a physical explanation. It is only a mathematical account. According to spacedynamics, the spacetime curvature is only an apparent property of spacetime induced by spacedynamics, which arises because of the mistaken assumption that the velocity of light is intrinsically constant and isotropic.

According to spacedynamics the accelerating expansion of the universe can be understood in terms of a dominant antigravitational interaction. Consider two well separated astronomical bodies of equal masses and that the associated velocity fields of the QS creating their gravitational fields are spinning round parallel axes. What will happen if the two bodies approximate eachother? In the case the velocity fields are spinning in the same sense, they will add up according to Equation (26), reinforcing the total velocity (gravitational) field, increasing the value of the (negative) gravitational potential and, on liberating a huge amount of energy, increase the binding energy of the system. However, in case the velocity fields are spinning in opposite senses their velocity fields will add up to zero and consequently cancel the individual negative gravitational potentials associated with each source without creating any binding energy. While the behavior in the first case is in conformity with the current gravitational theories, the second case is a novelty predicted by spacedynamics. According to conservation of energy, while in the first case kinetic energy can be created by letting the two bodies approximate each-other because they interact attractively, in the second case kinetic energy can be created by letting the bodies go away from each-other because they repel each other, they antigravitate.

Within the solar system the rotation axes of the solar and the planetary velocity fields are fairly well aligned and their velocity fields spin in the same sense as can be verified from the motions of planets round the Sun and of the satellites round the planets. An analogous polarization seems to be present within each galaxy. Within these systems only attractive gravitational interaction is present and is totally dominant. Nevertheless, the orientation (spin) of the different galaxies throughout the universe is almost random, which means that each galaxy and or galactic cluster most probably has oppositely rotating first nearest neighbor galaxies. Hence, on the overall repulsion (antigravitation) between galaxies and galactic clusters may still be dominant. This will say that from a large scale point of view the gravitational potential energy has a positive value, which is what is called dark energy. In fact sufficiently aligned galaxies (less than $90^{\circ}$ in their spins) still attract each other forming galactic clusters and super-clusters. Oppositely spinning galaxies and or galactic clusters repel each other, but such galactic clusters, although dominantly repelling each other, may still be connected by long chains of galaxies whose orientation gradually changes from one cluster to the other. This explains the observed filamentary large-scale structure observed in the universe of the galaxies. The spiral structure of galaxies apparently too has to do with such a chain effect however between clusters of stars.

Within the actual big-bang scenario it is possible to imagine that the expansion of the universe has stretched the de Broglie wave lengths of the elementary particles likewise it stretched those of the photons as is well known from the cosmic microwave background radiation. This may have reduced the momentum and the kinetic energy of the particles with respect to the QS in accord with the equation $(p=h / \lambda)$, allowing for the formation of atoms, molecules and larger aggregates able to support velocity (gravitational) fields of the QS oriented at random. Soon random gravitational and or antigravitational interactions between the aggregates could have started a segregation process. This way, a phase separation may have taken place along time in which the attractively interacting powders corresponding to given orientations of the spin of the velocity fields of the QS may have selectively been segregated and aggregated with others of compatible orientation into larger objects by attractive gravitation. During this phase separation process, the spin of the velocity fields is expected to be conserved so that the phase of randomly oriented velocity fields only changed in scale. This segregation/aggregation process may have evolved until asteroids, stars, galaxies and finally to the present stage of the randomly oriented galaxies and galactic clusters, in which antigravitation still is dominant.

\section{The Effects of the Gravitational Fields on the Propagation of Light and on the Rate of Clocks}

Many of the troubles, actually afflicting fundamental physics, were seeded by the wrong interpretation of the Michelson light anisotropy experiments. Within the view of spacedynamics the orbital and cosmic motion of Earth cannot give rise to relevant light anisotropy. However, the velocity field of the QS, generating the Earth's gravitational field certainly causes effects on the velocity of light and on the rate of clocks. Nevertheless, within the earthbased laboratories these effects are extremely small and therefore only highly sensitive experiments were able to detect the minuscule light anisotropy and time dilation effects predicted by spacedynamics. Fortunately synchronization of the GPS clocks is reaching to $0.1 \mathrm{~ns}$ (time for light to travel $3 \mathrm{~cm}$ ). With the help of these clocks several consistent observational facts show that 
the one way velocity of light is not isotropic. These clocks also have been able to evidence the gravitational time dilation. However, they also have evidenced that effects of the solar gravitational potential on the clocks moving with Earth are absent (please see Section 2). This proves that the gravitational time dilation is not due to the gravitational potential. In fact only motion with respect to the QS causes light anisotropy and time dilation effects.

In the view of spacedynamics, the one way velocity of light has a well defined value $c$ with respect to the QS and not with respect to all inertial references, as proposed in the TR. In the vacuum, the speed of light with respect to the QS is the same inside or outside gravitational fields. Hence, if the laboratory moves with respect to the QS or the QS moves through the laboratory with velocity $v_{0}$, the one way velocity of light with respect to the laboratory is:

$$
c_{e f f}=c+v_{0}
$$

Here the velocities $\boldsymbol{c}_{\text {eff }}$ and $\boldsymbol{c}$ are not those measured by the go-return roundtrip method, but are the one way speeds. Due to this effective velocity, an EM signal takes a longer time $(\Delta t)$ in a go-return roundtrip between two fixed points in a laboratory moving with respect to the QS than $\left(\Delta t_{0}\right)$ in a laboratory resting with respect to the QS:

$$
\begin{aligned}
& \Delta t=\Delta t_{0}\left[1-\left(v_{0} / c\right)^{2}\right]^{-1} \quad \text { (longitudinal) } \\
& \Delta t=\Delta t_{0}\left[1-\left(v_{0} / c\right)^{2}\right]^{-1 / 2} \quad \text { (transverse) }
\end{aligned}
$$

These simple and well known equations are at the origin of a large number of observed effects. All intermediary values are also possible for different directions. The anisotropy predicted by these equations is the one observed by the highly sensitive Michelson light anisotropy experiments. This effect also provides the genuine physical origin of time dilation because all the time standards by which clocks count time and physical processes in general run slower in a laboratory that is moving with respect to the QS.

If the laboratory is fixed within a gravitational field, created by a mass $M$, the QS moves through the laboratory with a velocity $v(r)=(\gamma M / r)^{1 / 2}$ along $+\phi$. In this case, the effective velocity of light within the laboratory is:

$$
\boldsymbol{c}_{e f f}=\boldsymbol{c}+(\gamma M / r)^{1 / 2} \boldsymbol{e}_{\phi}
$$

Hence, the duration of go-return light roundtrips within a gravitational field are given by:

$$
\begin{aligned}
& \Delta t=\Delta t_{0}\left[1-U / c^{2}\right]^{-1} \quad \text { (longitudinal) } \\
& \Delta t=\Delta t_{0}\left[1-U / c^{2}\right]^{-1 / 2} \quad(\text { transverse })
\end{aligned}
$$

where $U=V^{2}=(\gamma M / r)$ and $\Delta t_{0}$ is the frequency outside the gravitational field. The corresponding frequencies are:

$$
\begin{aligned}
& f=f_{0}\left[1-U / c^{2}\right] \quad \text { (longitudinal) } \\
& f=f_{0}\left[1-U / c^{2}\right]^{1 / 2} \quad \text { (transverse) }
\end{aligned}
$$

The total time delay accumulated in go-return roundtrips along large distances within a gravitational field must be obtained by integration.

According to Equations (33) up to (37), motion with respect to the QS is the unified cause of the gravitational time dilation, the gravitational spectral redshifts, the increased life time of speeding Muons, the redshift of radiation emitted by speeding atoms, the light anisotropies as well as the excess time delays of radar signals within the solar system. They express the genuine physical origin of the excess time delay in light roundtrips and radar signals within the solar system as well as of the augmented time periods of the electromagnetic oscillations of all radiation sources and time standards. These time dilation effects are ruled by conservation of the total energy of the system, which involves rearrangement of the quantized energy level structure of the radiation sources. This rearrangement responds the same way to motion of the laboratory with respect to the resting QS or to motion of the QS through the resting laboratory. This is why Equations (33) and (35) are so similar. It also is true that motion of a radiation source with respect to the QS increases the mass involved in the EM oscillations thereby reducing the frequency.

If it were true that the lengths of material bodies contract in the direction of their motion with respect to the QS according to the Lorentz-Fitzgerald length contraction, then in the Michelson light anisotropy experiments the longitudinal go-return roundtrip time would become equal to that of the transverse go-return roundtrip, so that the anisotropy measured with the Michelson interferometer would cancel in spite of the velocity of light being anisotropic. However, the observation of light anisotropy by the most sensitive conventional Michelson experiments, which has been confirmed by measurements of the one way velocity of light, indicate that this length contraction does not occur. Another point that comes into question is if motion with respect to the QS does distort the quantized energy level structure of real radiation sources (atoms, molecules etc.) so that the frequency (energy) of the radiation emitted by such anisotropic atoms would depend on the direction with respect to the motion. If true, such anisotropy would have been detected already in the Ives-Stilwell experiment [4]. To now no experimental evidence of such anisotropy in free atoms has been found and therefore it must be excluded. This point concerns not only atomic sources but also all 
strongly confined radiation systems. If the frequency (energy) of the radiation emitted by atoms that supplies the laser cavities is isotropic why then would the radiation emitted by these cavities be anisotropic? Only in the measure that such cavities do not depend too much on the feedback supplied by the atomic sources can they disclose anisotropy effects. This is almost certainly the reason why some light anisotropy experiments using directly laser cavities or other strongly confined radiation systems gave null results. The negative result of anisotropy experiments like that by Brillet and Hall [42], which is often cited as the most sensitive light anisotropy experiment, is in fact a negative result for the LorentzFitzgerald contraction.

Due to the effective velocity of light (Equation (35)), the velocity of light measured by the go-return roundtrip method within a laboratory fixed within a gravitational field seems lower from the viewpoint of an external observer. However, the rate of all clocks (time standards) within the gravitational field is reduced in the same proportion as compared with external clocks. Because of this simple and quite obvious fact a local observer, using the go-return light roundtrip method, finds the same (proper) value for the velocity of light inside or outside the gravitational field. On the other hand, an external observer measuring distances within the gravitational field by the method of go-return light roundtrips, without taking into account the effective velocity of light (Equaton (35)), will obtain the non-Euclidean metric underlying Einstein's spacetime curvature (please see Section 10.3 for details). It hence is clear that this spacetime curvature is simulated by spacedynamics and hence is apparent. The spacetime curvature arises because of the inappropriate measuring method and the mistaken assumption in the TR that the velocity of light is intrinsically isotropic and constant.

\section{The Predictions of Spacedynamics and the Experimental Observations}

\subsection{Michelson Light Anisotropy Experiments}

The Michelson interferometer (see Figure 11) measures the anisotropy of light by the displacement of the interference fringes when the interferometer is rotated. Hence, if the interferometer does not rotate, it measures nothing. In fact what the Michelson interferometer measures is its velocity with respect to the medium (QS) that propagates light. In many Michelson experiments [7,8], frequently reputed as the most reliable ones, the interferometer is fixed within a vacuum case and does not rotate in the earthbased laboratory. It hence cannot detect the anisotropy due to the local velocity field of the QS round Earth. Such interferometers however rotate with Earth and so can measure the anisotropy of light due to the orbital and cosmic motion of Earth, which is the motion of Earth with respect to the QS. However, in the view of spacedynamics, this velocity is much too low to be detected by these experiments.

In the view of spacedynamics, the only motion, that can cause a significant genuine anisotropy of light within the earthbased laboratories, is the one due to the velocity field of the QS round the Earth in the sense of the Moon's orbital motion, which generates the gravitational field of Earth itself. This velocity field reaches $7.9 \mathrm{~km} / \mathrm{sec}$ on surface. While Earth is commoving with the QS round the Sun, round the galactic center etc., it rotates much too slowly (only $0.46 \mathrm{~km} / \mathrm{sec}$ at the equator) to bring the earthbased laboratories to rest with respect to the QS. At the sites of the experiments the residual velocity is $\sim 7.5$ $\mathrm{km} / \mathrm{sec}$, the direction of which is closely fixed with respect to the earthbased laboratories and is constant the whole day and the whole year. Many and the most precise Michelson interferometers were constructed along nearly 40 years in the USA, first by Michelson-Morley [11] then by Morley-Miller and finally by Miller alone [12]. The obtained anisotropy data all fall within the range from 6 to $12 \mathrm{~km} / \mathrm{sec}$. However, the most precise and systematic data were obtained by Miller in his late non-stop (day and night) 1925-1926 anisotropy experiments [12]. The data were integrated over many weeks and plotted hour per hour for the 24 hours of the day and for four different epochs of the year. These data confirm very closely the predictions of spacedynamics qualitatively and quantitatively, see Figure 12. They also have been closely corroborated by measurements of the one way velocity of light with the help of the GPS [3]. Nevertheless, as these data are nearly two orders of magnitude smaller than expected from the ether theories, they usually have been attributed to spurious effects [43] or simply ignored.

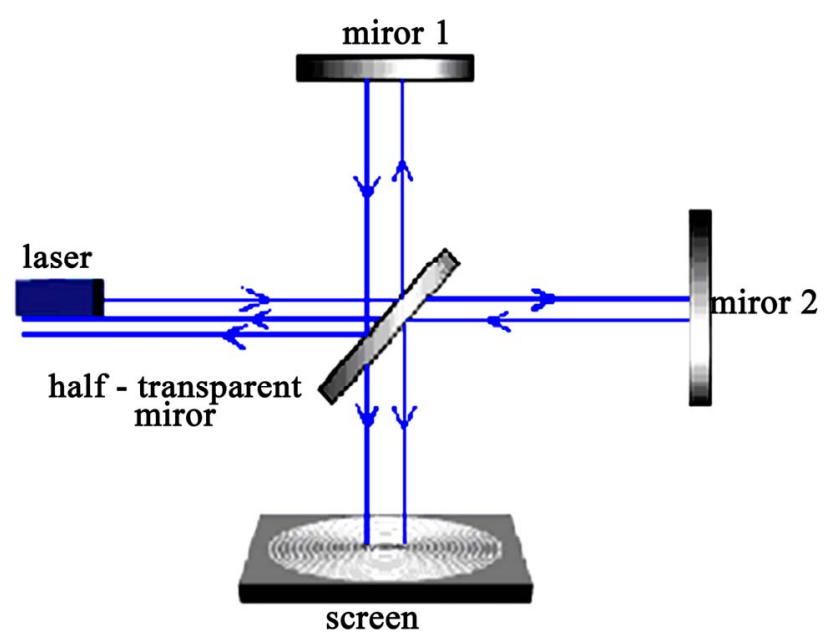

Figure 11. The Michelson interferometer and light anisotropy experiments. 


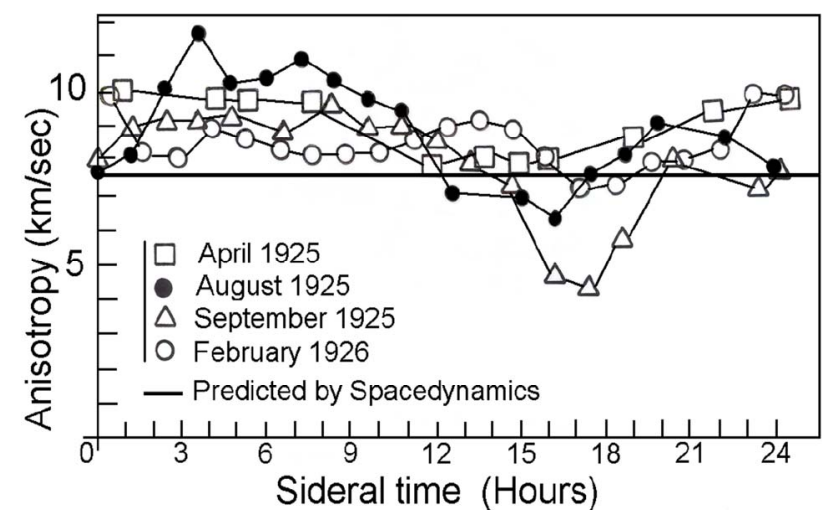

Figure 12. Ultimate light anisotropy data obtained by $D$. Miller. The small but systematic variations along the 24 hours may be due to spurious causes.

In another light anisotropy experiment, performed in the laboratories of the MIT [13], two masers were optically coupled at 90 degrees on a stable turntable, which is equivalent to a Michelson interferometer. On turning the apparatus about a vertical axis, a positive anisotropy of similar magnitude as that measured by Miller [12] was observed. The anisotropy of about $8 \mathrm{~km} / \mathrm{sec}$ had a fixed direction with respect to the laboratory. However, because of the smallness and the fixed direction of this anisotropy, it was attributed to magnetostriction of the constantan mirror spacers caused by the Earth's magnetic field that is of about 0.3 Oe. This however has neither been checked nor confirmed. The value and sign of magnetostriction of constantan for such low fields is not known.

Another light anisotropy experiment similar to those by Morley and Miller, but searching for effects of the orbital and cosmic motion of Earth, Joos [44] obtained a null result as predicted by Spacedynamics. However, for individual turns of the interferometer, the data are not inconsistent with the predictions of Spacedynamics and with Miller's data. Several anisotropy experiments using Mössbauer effect [9] and lasers [42] were performed too. While the Mössbauer anisotropy experiment gave a null result because of proven flaws in their conception [10] the anisotropy experiment with the servo stabilized $\mathrm{He}-\mathrm{Ne}$ laser having its frequency locked to a Fabry-Perot resonator apparently was constrained to give null light anisotropy. The result of this experiment is in fact a null result for the Lorentz-Fitzgerald contraction. The simple fact that several Michelson light anisotropy experiments gave positive results already shows that the usually alleged Lorentz-Fitzgerald contraction of the interferometer arms is absent. In reality direct experimental evidence of the Lorentz-Fitzgerald contraction never has been found. All the alleged evidences in the literature are indirect and have been introduced to safeguard theoretical views.

\subsection{Gravitational Time Dilation and Redshifts}

Spectral redshifts and gravitational time dilation are now well confirmed experimentally [45-47]. The increase of the periods of the time standards and the consequent decrease of the frequency of classical and quantum mechanical oscillators (molecules, atoms, nuclei etc.) during transitions between quantum states and the emission of radiation is simply due to the fact that matter waves need a longer time to complete an oscillation roundtrip period when the laboratory moves with respect to the QS Equation (34) or the QS moves through the laboratory Equation (36). This provides the transparent and genuine physical origin of the observed time dilation, spectral redshift and light anisotropies. An associated rearrangement of the energy level structure of the radiation sources may occur. Although the TR predicts these effects in terms of the hypothetical geometry of spacetime, it does not bring to light the genuine physical cause of the gravitational time dilation and the spectral redshifts.

\subsection{Excess Time Delay of Radar Signals within the Solar System}

Taking into account the effective signal velocity within the velocity field of the Sun stipulated by Equation (35), the excess time delay was calculated using a simple but precise numerical integration method. The method consisted in dividing the straight-line paths to Venus and the path back to Earth for the different impact parameters $(R)$ into a large number of segments, calculating the effects of the average transverse and of the parallel velocity of the QS in favor or against the signal velocity along each segment and finally adding up the affects along the whole roundtrip.

In the calculation, the positions of Earth and Venus as well as the different signal path due to the motion of Earth during the roundtrip was considered (see Figure 13). However, the ellipticity of the orbits was not taken into account. The result shows that, although the signal velocity in the Earth-Venus and the Venus-Earth journeys is considerably asymmetric, the effective excess time delay $\Delta t_{\text {eff }}$ for a full roundtrip is almost exactly the one found in the experiments by Shapiro [48]. Figure 14 shows the excess time delays (positive) or time gain (negative) of the radar signals in milliseconds (ms) as a function of the solar impact parameter $R$ for the go to Venus and the back to Earth journeys, before and after superior conjunction.

Figure 15 displays the effective excess time delays for full go-return roundtrips in microseconds ( $\mu \mathrm{s})$ as a function of the closest approach $(R)$ from the Sun, before and after superior conjunction (see last column in Table 1). The excess time-delay data $\Delta t_{\text {eff }}$, predicted by spacedynamics, are in good qualitative and quantitative agreement 
with the time-delay data measured by Shapiro [48] and displayed in Figure 16. If the ellipticity of the orbits had been considered, this agreement would even be better. In spacedynamics, the excess time delay arises from a genuine physical effect. It is due to the increase of the effective travel time as given by Equation (36) due to the effective velocity within the velocity field of the QS generating the inertial dynamics within the solar gravitational field and not to the increase of the geometrical distance due to the spacetime curvature as considered in GR. Other even more precise measurements of the excess time delay (Shapiro Effect) of signals passing by the Sun have been made with the help of Mars landed transponders during the Mariner 6 and Mariner 7 missions as well as other spacecrafts (Cassini). However, in all of these experiments what was measured is the effective excess time delay for complete go-return signal roundtrips. No excess time delay data are available for one way travels.

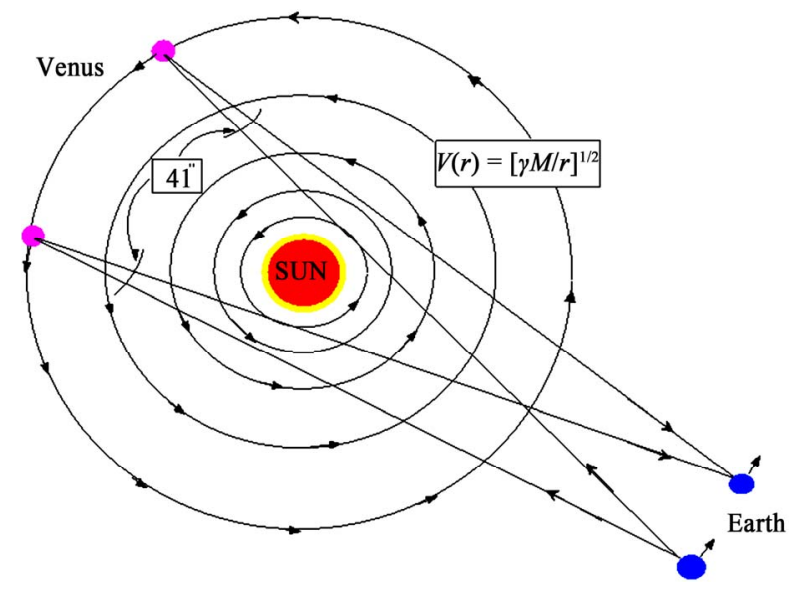

Figure 13. The path of radar signals from Earth to Venus and back to Earth within the velocity field of the sun, before and after superior conjunction.

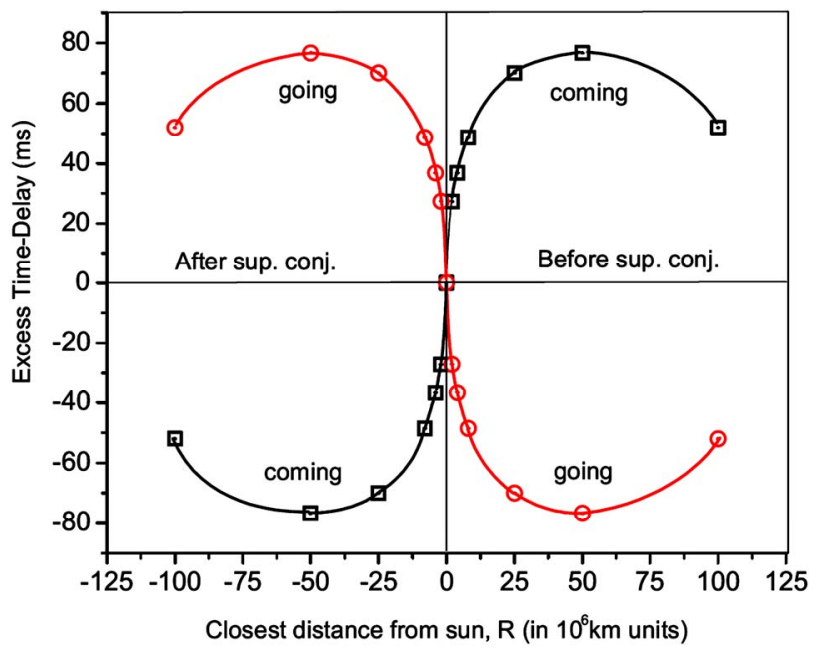

Figure 14. The excess time delay $(+)$ and the time gain $(-)$ of radar signals before and after superior conjunction.

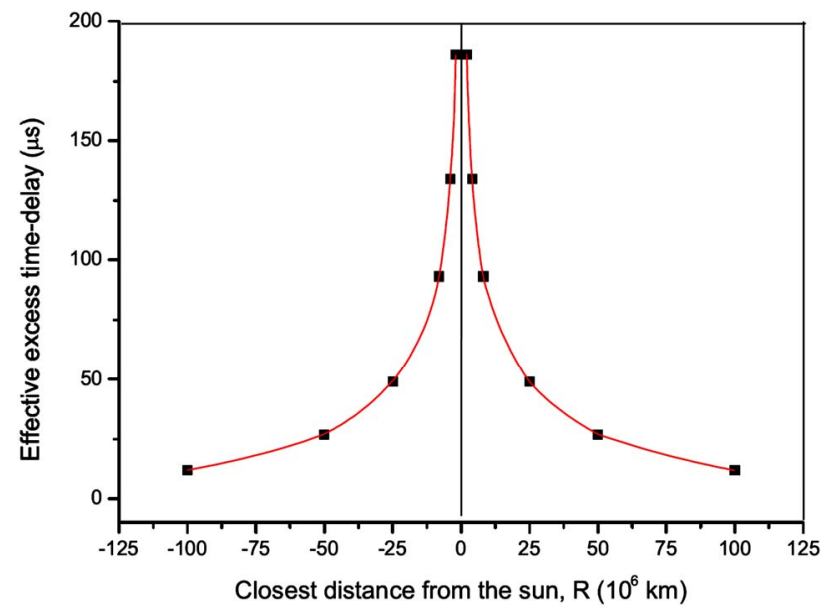

Figure 15. The effective time delay for a complete go-return travel as calculated from spacedynamics, please see fourth column in Table 1. The value of $R= \pm 100 \times 10^{6} \mathrm{~km}$ in Figure 15 corresponds to about \pm 180 days in Figure 16 .

Table 1. The Excess time-delays for individual go and return journeys and the effective excess time-delays (last column) for full go-return roundtrips of radar signals between Earth and Venus for impact parameters $R=0,2,4,8,25,50$ and 100 million $\mathbf{k m}$ from the center of the Sun before and after superior conjunction. The time-delay for $R=0$ is very small because along this path the velocity field has no parallel component along the whole signal path. The steep inflection at $\boldsymbol{R} \sim \boldsymbol{0}$ could have been observed by Shapiro if the radar signal path had passed above or below the sun.

\begin{tabular}{cccc}
\hline$R\left(10^{6} \mathrm{~km}\right)$ & go: $\Delta t(\mathrm{~ms})$ & Return: $\Delta t(\mathrm{~ms})$ & $\Delta t_{\text {eff }}(\mu \mathrm{s})$ \\
\hline 100 & -51.987 & 51.999 & 12 \\
50 & -76.697 & 76.725 & 28 \\
25 & -70.007 & 70.056 & 49 \\
8 & -48.585 & 48.679 & 94 \\
4 & -36.699 & 36.833 & 134 \\
2 & -27.165 & 27.351 & 186 \\
0 & 0.035 & 0.035 & 70 \\
-2 & 27.351 & -27.165 & 186 \\
-4 & 36.833 & -36.699 & 134 \\
-8 & 48.679 & -48.585 & 94 \\
-25 & 70.056 & -70.007 & 49 \\
-50 & 76.725 & -76.697 & 28 \\
-100 & 51.999 & -51.987 & 12 \\
\hline
\end{tabular}

The calculated excess time delay data in the second and third column in Table 1 are for one way travels. The fact that spacedynamics predicts correctly the effective excess time delay (Shapiro effect) confirms that the velocity field Equation (1) simulates correctly Einstein's spacetime curvature. However, besides this, it also shows that GR does not provide the exact instant at which the radar signal is reflected by Venus. 


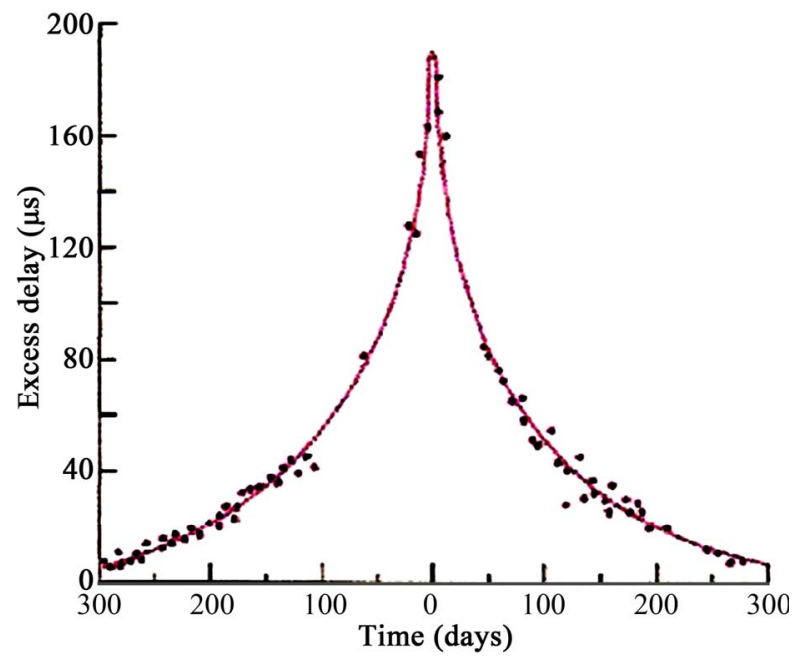

Figure 16. The effective time delay for a complete go-return travel as measured by Shapiro [48].

\subsection{Anomalous Red/Blue Shifts}

Astronomical observations show plenty of red/blue shifts of radiation of non-Doppler origin. They are especially significant for binary stars or binary galaxies that orbit round the center of mass about an axis perpendicular to the line of sight. These frequency shifts are several orders of magnitude larger than can be explained by GR. To now their exact origin is unknown [17]. From the viewpoint of spacedynamics, these frequency shifts are simply caused by the variable excess time delay $\Delta t$ of the radiation by the interposition and motion of the velocity fields of the QS due to very massive bodies. They are essentially proportional to $[\mathrm{d}(\Delta t) / \mathrm{d} R] \mathrm{d} R / \mathrm{d} t$, where $R$ is the impact parameter of the radiation passing by the companion star or galaxy or by any other strong gravitational source. If the velocity $\mathrm{d} R / \mathrm{d} t$ is zero, the effect vanishes as it must. Variable excess time delays for individual one way travels are certainly present in the frequency shifts, observed when Pioneer 6, (operating at $2295 \mathrm{MHz}$ ) passed by the opposite side of the Sun during superior conjunction $[17,18]$. Such frequency shifts may also be present in the signals from Mars during its passage behind the Sun. However, while in the case of Pioneer 6 the predicted frequency shift is a nearly even blue-shift achieving about $200 \mathrm{~Hz}$ in the region of low impact parameters $[R= \pm 10$ solar radii], in the case of the Mars Lander operating at the same frequency a redshift achieving about $260 \mathrm{~Hz}$ for low $R$ may be observed. Effectively, in Goldstein's data for Pioneer 6 [18], see also Merat et al. [17] a positive frequency shift is observed. Nevertheless, the very significant Doppler corrections, due to the orbital motion and rotation of Earth, the effect due to variation of the excess time delay caused by the solar velocity field cannot be evaluated with security. Moreover, the depression of the frequency in the immediate vicinity of the Sun apparently is the Doppler shift, due to the motion of Earth in the EarthMoon system that has not been considered in the corrections by Goldstein.

\subsection{Effects of the Velocity Field of the QS Equation (1) on the Clocks}

The effect of the velocity field Equation (1) on the clock rate is dictated by Equations (34) and (36). Accordingly, clocks in direct circular equatorial orbits round an astronomical body, which itself normally is commoving with the QS in the velocity field of a larger body, are locally resting with respect to the QS and consequently these clocks go not slow. Such clocks are all naturally synchronous throughout the universe. Nevertheless, the rate of clocks, fixed within gravitational fields, is reduced according to Equations (36). It is interesting to note here that if the satellites of the GRACE project [3] had been moving in direct circular equatorial orbits round Earth, then the one way velocity of light would not have shown the anisotropy of $8 \mathrm{~km} / \mathrm{sec}$ but would have shown a null result.

\subsection{Setting the Clocks of the GPS Satellites before Launch}

The orbits of the GPS satellites are inclined with respect to the equator by angles between $55^{\circ}$ and $63^{\circ}$. Therefore they have a velocity component with respect to the QS $v_{o}(1-\cos \alpha)$ along $-\phi$ and $v_{o} \sin \alpha$ along $\pm \theta$ where $v_{o}=3.87 \mathrm{~km} / \mathrm{sec}$ and $\alpha$ is the angle of $v_{o}$ with respect to the equator or parallels. The effective velocity is $v_{o}[2(1-\cos \alpha)]^{1 / 2}$ and the estimated average velocity of the GPS satellites over the entire orbit is $0.8 v_{o}$. Considering moreover the velocity of $0.22 \mathrm{~km} / \mathrm{sec}$ of the $C s$ atoms in the atomic clocks, this makes $3.30 \mathrm{~km} / \mathrm{sec}$. Analogously, for the earthbased stations we find 7.20 $\mathrm{km} / \mathrm{sec}$. From this we find that the rate of the GPS clocks must be slowed by $4.5 \times 10^{-10} \mathrm{sec} / \mathrm{sec}$ before launch in order to run closely synchronous with the clocks resting on Earth when in orbit. This is exactly the value calculated by the GPS staff for NASA [47]. They however had to consider many incoherent terms for GR and SR.

\subsection{Absence of Effects of the Solar Gravitational Potential on the Terrestrial and GPS Clocks}

It has been verified that the solar gravitational potential does not affect the rate of terrestrial and GPS clocks $[5,6]$. This observation is known as the noon/midnight problem and to now it is unsolved. In spacedynamics this observational fact is easily and straightforwardly explained in terms of Equations (34) and (36). Earth moves along with the QS in the velocity field generating the gravita- 
tional field of the Sun and hence its velocity with respect to the QS is closely zero $\left(v_{o}=0\right)$. This while implying absence of light anisotropy effects due to orbital and cosmic motions of Earth, as evidenced by Michelson light anisotropy experiments, also is the obvious reason why the velocity field (gravitational potential) of the Sun does not affect the rate of the earthbased and GPS clocks. This fact proves that gravitational time dilation and slowing of the clock rates is not caused by the gravitational potential as asserted by GR but is caused by motion of the clocks with respect to the QS or motion of the QS through the resting clocks. Hence, the rates of identical clocks one on Mars and another one on Earth may not show effects of the solar gravitational potential but only the effects of the local gravitational fields of respectively Mars and Earth. This in fact is valid for all the planets of the solar system. Present day technology is perfectly capable of testing these predictions.

\subsection{Scandalous Clocks or Real Disparity in the Arrival of the Pulsar Signals}

The resolution of optical and radio astronomy observations can be improved by orders of magnitude by interferometric methods of observations made in separate laboratories. The condition is that these observations be synchronous so that the data simultaneously recorded in separate observatories can be superposed with the help of computers. For Very Long Baseline Interferometry (VLBI) radio astronomy observations clock synchronizations achieve $0.1 \mathrm{~ns}$. Nevertheless, on confronting the so synchronized clocks with the arrival of the extremely precise ticks of distant pulsars, which according to the TR are synchronous, enormous discrepancies of up to $4.2 \mu \mathrm{s}$ have been verified for antennas at the edges of the earthglobe along the Earth's orbital motion. However, along the transverse direction the arrival is synchronous $[5,6]$. This is again raising hot debates about the nature of space as asserted by the different theories. While some people speak of scandalous clocks that are biased along the Earth's orbital motion [5], others see in these facts definitive prove that the velocity of light is not the same along different directions within the solar system [14].

From the viewpoint of spacedynamics there is no surprise at all. The wave fronts of a signal coming from a distant pulsar toward the Sun are rotated at a time rate given by Equation (4a). The wave fronts are refracted and the propagation direction is slanted toward $-\phi$ by a total angle $\beta$ so that $\sin \beta=V / c$, which is equal to the usual aberration angle of light. The signal would go along the path $c$ as shown in Figure 17. However, at the same time the signal is dragged by the velocity field along $+\phi$ without changing the slope of the wave fronts. The effects of refraction and of drag compensate each other exactly, so that the signal path goes effectively along the radial path $c^{\prime}$, see Figure 17. The signal velocity along this radial direction is lower, given by $c^{\prime}=\left(c^{2}-V^{2}\right)^{1 / 2}$, where $V$ is given by Equation (1). In GR this lowered velocity of light along the radial coordinate is imputed to stretching of the radial coordinate due to spacetime curvature. In spacedynamics it is due to the fact that, in order to the signal to go along the radial coordinate, it necessarily must develop a component along $-\phi$. In optical observations from Earth the telescope must be tilted pointing along $c\left(r_{o}\right)$, along which the wave fronts are perpendicular to the path of light and the velocity of light with respect to the telescope is exactly the usual velocity of light $c$. This is the well known aberration of stars.

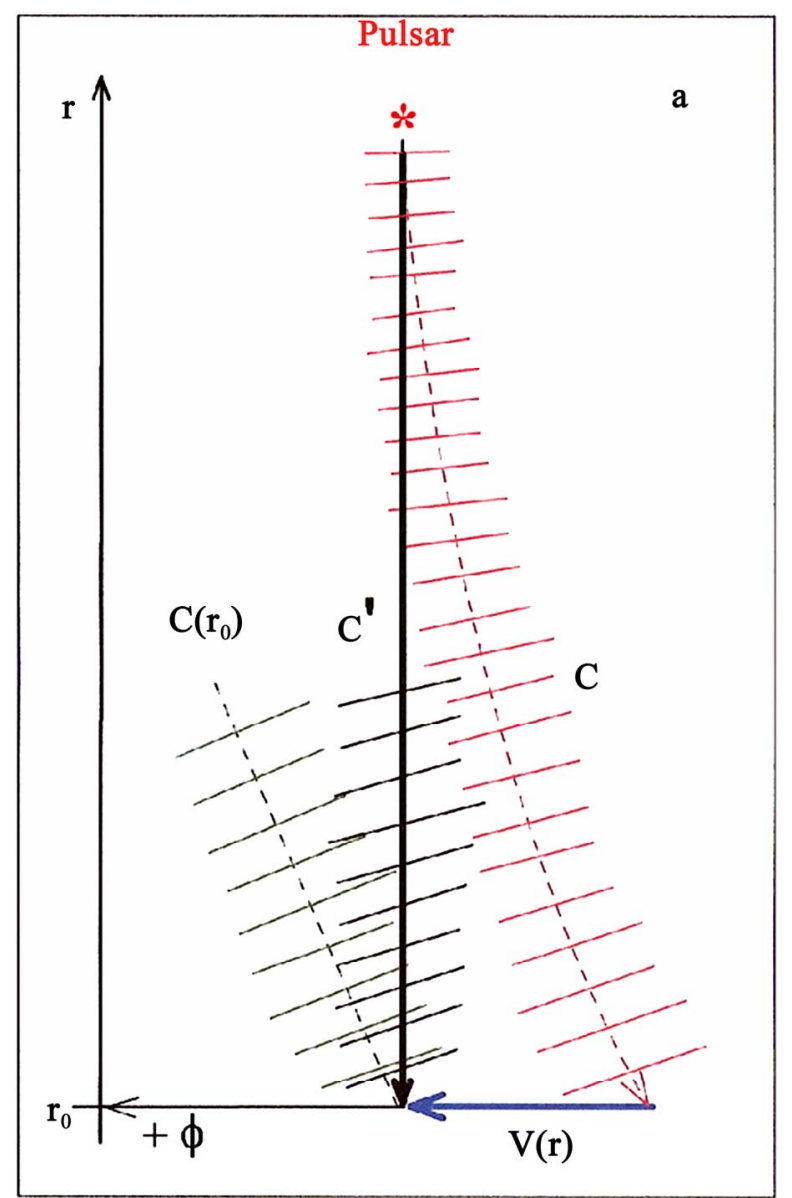

Figure 17. A light or EM signal from distant pulsar propagating into the solar velocity field directly toward the Sun. The signal under the refraction rate, given by Equation (4a), would go along the path $c$. However, drag by the solar velocity field shifts the signal back to the radial path $c^{\prime}$ $=\left(c^{2}-V^{2}\right)^{1 / 2}$, without changing the slope of the wave fronts. In optical (midnight) observations from Earth, the telescope must point along $c\left(r_{o}\right)$ along which the wave fronts of light are perpendicular to the signal path and the velocity with respect to the telescope is exactly the usual velocity of light (c). In the figure, the effects are largely exaggerated to make them visible. 
The arrival of the pulsar signal along $c^{\prime}$ in stations $L_{1}$ and $L_{2}$ equidistant from the pulsar, fixed in the solar non-rotating reference at the Earth's orbital radius and separated by the diameter of Earth, will not be simultaneous as shown in Figure 18. A given wave front reaches $L_{1}$ up to $4.2 \mu$ s before $L_{2}$ because the wave fronts are refracted as dictated by Equation (4a). Moving $L_{1}$ and $L_{2}$ along the $L_{1}-L_{2}$ direction will not change the situation. Hence, the non-synchronous arrival of the signal to the different and equidistant earthbased observatories is real and shows that synchronization of the clocks in the distant observatories with the help of the GPS was not incorrect. The fact that the arrival of the pulsar signal is out of synchrony by $4.2 \mu$ s along the Earth's orbital motion and is synchronous along the direction transverse to the orbital motion exactly as dictated by Equations (4) is a spectacular confirmation of the predictions of spacedynamics.

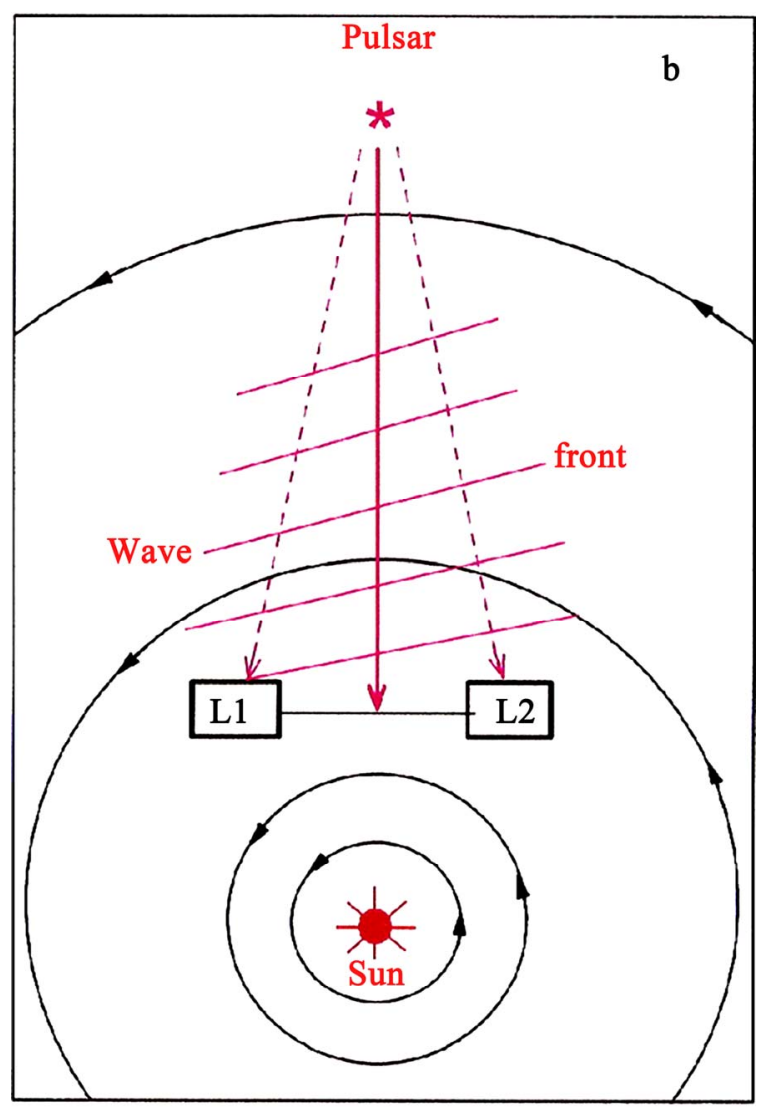

Figure 18. The wave fronts of an $E M$ signal coming from a distant pulsar toward the Sun along $c^{\prime}$ are slanted due to the refraction by the solar velocity field Equation (4a) as also shown in Figure 17. The signals are detected in two stations fixed in the solar gravitational field at a distance of the earth's orbit and equidistant from the pulsar. The wave front reaches first the station $L_{1}$ and only $4.2 \mu$ s later $L_{2}$. The situation is not changed by motions along the $L_{1}-L_{2}$ direction. All effects are largely exaggerated in the figure to make them visible.

\subsection{The Astronomical Motions Closely Track the Motion of the QS}

The nearly circular planetary orbits are squeezed into the disc shaped solar system. Analogously the stellar orbits are squeezed into the galactic disc. Why? From the spacedynamics viewpoint all these bodies minimize their velocity with respect to the QS. This reduction of velocities may have taken place during the expansion of the universe that stretched the wavelengths $(\lambda)$ of the particles analogously as it stretched that of the photons, as is well known from the cosmic background microwave radiation. Stretching and shrinking of the wavelengths affects the velocity of particles according to de Broglie's equation $(p=m v=h / \lambda)$. Orbital motions of the planets likewise motion of the QS (gravitational fields) may have been set up during formation of the planets, stars and galaxies. All the experiments that searched for the velocity of Earth with respect to the medium propagating light found much too low results. This absolutely corroborates the viewpoint of spacedynamics.

\subsection{The Gravitational Deflection of Light}

The velocity of particles with a rest mass normally is much lower than the velocity of light. Therefore they follow perfectly well the refraction rates expressed by Equations (4) as shown in the previous sections. Contrarily, light always propagates with the maximum velocity with respect to the QS. Hence, the refraction rate dictated by Equations (4) can act freely on one component only when the other component is negligibly small. When a light beam propagates through the velocity field of the Sun, its $r$ and the $\phi$ velocity components cannot always be refracted freely. A light beam directly incident toward the Sun has practically only a velocity component along $r$ and is refracted according to Equation (4a). Therefore it gains a velocity component along $-\phi$ and hence reduces its radial velocity component to $c^{\prime}=\left(c^{2}-V^{2}\right)^{1 / 2}$ where $V$ is the velocity of the QS as shown in Fig.19. GR attributes this reduction to stretching of the radial distances due to the spacetime curvature.

Consider two light beams, propagating toward the Sun along parallel rectilinear paths by equidistant and opposite sides of the Sun as shown in Figure 19. First the wave vectors will be refracted according to Equation (4a) by a total angle $+\alpha$. Near to the Sun, where the solar velocity field achieves $436 \mathrm{~km} / \mathrm{sec}$, refraction by Equation (4b) dominates and the wave vector is refracted oppositely by $-2 \alpha$. However, the solar velocity field is favorable to the prograde ray so that it propagates less time near the Sun and hence is deflected by only an angle $-(2 \alpha-\delta)$. To the retrograde ray the solar velocity field is unfavorable and hence it takes a longer time near the Sun and hence is refracted be a larger angle $-(2 \alpha+\delta)$. 
Finally, after passing by the Sun and going away at the opposite side, both beams deviate back according to Equation (4a) by nearly an angle $+\alpha$. The differentiated refraction $\delta$ by Equation (4b) causes the convergence of the rays that is responsible for the light lensing effect by the gravitational fields. The value of the effective deflection of light by the solar gravitational field can be calculated simply by multiplying the rate of refraction Equation (4b) times the excess time delays listed in Table 1 . On the solar surface $\left(\mathrm{R}=6.9565 \times 10^{8}\right)$ the refraction rate is $W_{\phi} \sim 0.0359 \mathrm{deg} / \mathrm{sec}$ and interpolating the value of $\Delta t$ in Table 1 for light passing by the surface of the Sun, the total excess time delay for a light pulse passing by the retrograde side (or the gain at the prograde side) gives about $13.5 \mathrm{~ms}$. The product $W_{\phi} \times \Delta t$ gives $\delta=1.745 \mathrm{arc} / \mathrm{sec}$, which is about the observed value.

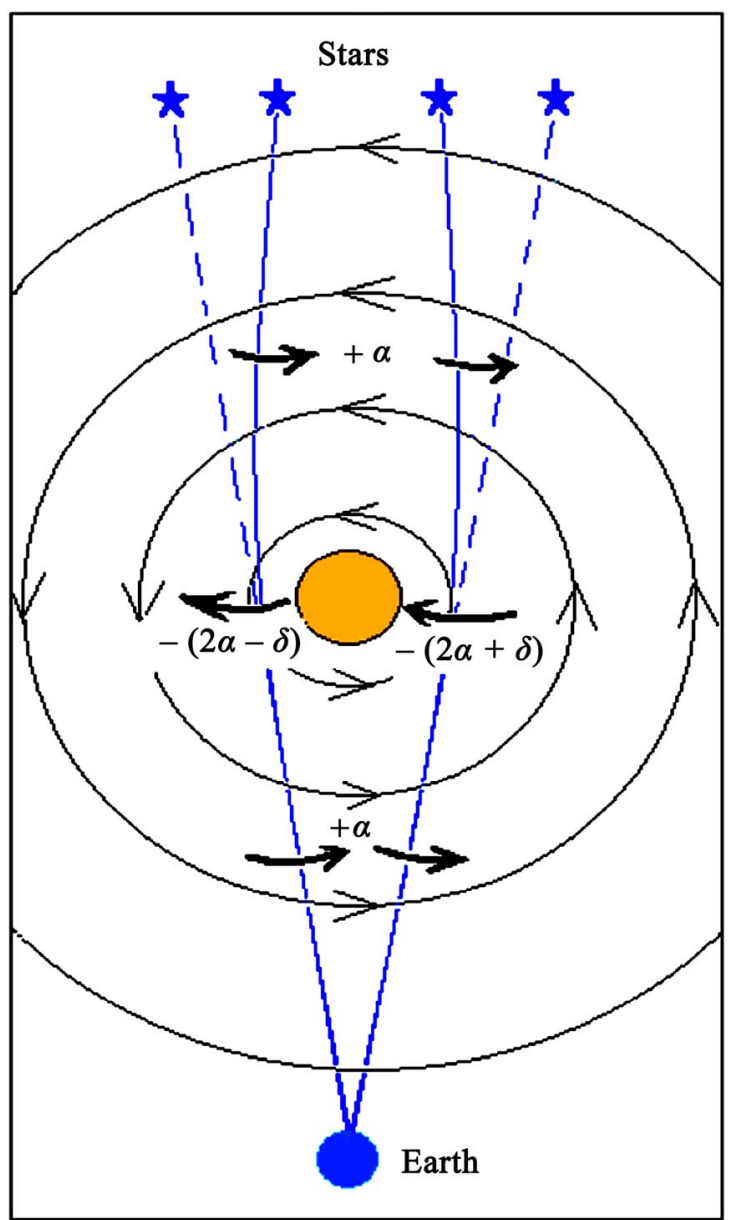

Figure 19. Sketch of the light lensing effect by a heavy mass, caused by differentiated refraction according to Equations (4). First a rotation $+\alpha$ is caused by Equation (4a). Near the Sun Equation (4b) dominates and rotates the ray oppositely by $-(2 \alpha \pm \delta)$ and finally, on moving away from the Sun, another rotation $\sim+\alpha$ takes place due to Equation (4a). The convergence of llight results from the excessive deflection $\delta$ at the retrograde side and the insufficient deflection $\delta$ at the prograde side.

\subsection{The Perihelion Precession}

An analogous differentiated refraction of the propagation velocity $\boldsymbol{v}_{\text {prop }}$ of an orbiting body as in the preceding sub-Section 10-10 must be responsible for the perihelion precession of elliptical orbits. At the aphelion the direction of $\boldsymbol{v}_{\text {prop }}$ is opposite to the velocity field as well as to the orbital motion (see Figure 5), thereby increasing the time of permanence in this region of the orbit. Hence, $\boldsymbol{v}_{\text {prop }}$ refracts during a longer time. At the perihelion $\boldsymbol{v}_{\text {prop }}$ is parallel to the velocity field pointing in the prograde sense thereby displacing the particle more rapidly in the orbit. Therefore $\boldsymbol{v}_{\text {prop }}$ has not time enough to recover the tangential direction. It recovers it only somewhat beyond the ideal perihelion point of the orbit. In this way the perihelion is displaced a little bit in the prograde sense in each orbital roundtrip.

\subsection{The Conventional Relativistic Effects can Easily be Reinterpreted as Effects Due to Motion with Respect to the QS}

If Earth rests with respect to the QS, as demonstrated by so many experimental facts, then the very high velocities of elementary particles and or atoms within earthbased laboratories are equally high velocities with respect to the local QS. In the view of spacedynamics, the solar and galactic velocity fields are not effective within the earthbased laboratories because Earth is commoving with the QS in these velocity (gravitational) fields. The velocity field of the QS that flows through the earthbased laboratories is only the one creating the local gravitational field of Earth itself. On the surface of the rotating Earth this velocity varies from $V=7.4 \mathrm{~km} / \mathrm{sec}$ at the equator to $V=7.91 \mathrm{~km} / \mathrm{sec}$ round the poles. This is a rather low velocity compared with the velocity of light. To first approximation the effects of this velocity are proportional to $V^{2} / c^{2}$, which is in the order of $10^{-10}$. Therefore it is impossible to say if the so called relativistic effects on the hydrogen radiation in the Ives-Stillwell experiment [4] $\left(v \sim 10^{3} \mathrm{~km} / \mathrm{sec}\right)$, the increased lifetimes of Muons in cosmic rays $(v \sim c)$, the relativistic mass of electrons and protons from particle accelerators $(v \sim c)$ or the relativistic energy term in atomic and nuclear states are due to the relative velocity or to the velocity with respect to the QS. In reality these so called relativistic effects can easily be reinterpreted as effects due to the velocity with respect to the QS. The usual Lorentz transformation is to be seen as the viewpoint of the laboratory observer using the improper go-return light roundtrip measuring method, on converting his observations from a reference $K$, resting with respect to the QS (closely the situation of the earthbased laboratories), to a reference $K^{\prime}$ moving with respect to the QS. Also the universality of the laws of physics throughout the universe, the ap- 
parent covariance of the variables involved in the laws of physics observed throughout the universe, must be imputed to the fact that the natural astronomical bodies all very nearly rest locally with respect to the QS. Only within laboratories, moving at high speed with respect to the QS or fixed deeply within strong gravitational fields, could these laws be significantly affected. If in fact such high velocities affect the laws of physics or not, will be settled only when humans be able to test this under really high velocity.

\subsection{Black Holes}

In very strong gravitational fields, in which the velocity of the QS (Equation (1)) reaches values equal or larger than the velocity of light $(c)$, the black hole phenomenon takes place. Note that motion of the QS at velocities larger than $c$ does not violate any principle of physics because the QS is the ultimate reference for motions and hence motion of the QS is gauge invariant. The "horizon" of a black-hole is the limit up from which light can still escape and below which it becomes confined. Within a laboratory, fixed below the horizon, several unusual effects may take place: The rate of clocks fixed within the singularity falls to zero, light cannot move anymore toward $-\phi$ but only into a limited solid angular sector toward $+\phi$. According to spacedynamics, likewise the velocity of light is anisotropic within a gravitational field, the horizon too is anisotropic. While for light propagating toward $-\phi$ the horizon is fixed by

$$
V(r)=[\gamma M / r]^{1 / 2}=c,
$$

for light propagating along $+\phi$ it is fixed by

$$
V(r)=[\gamma M / r]^{1 / 2}=\sqrt{2} c .
$$

\subsection{The Twin Paradox}

The famous twin paradox arises because of the reciprocal symmetry of the measurements made by relatively moving observers as asserted in the TR. Einstein, Born and Möller as well as others have claimed that specifically in the case of the twin paradox the reciprocal symmetry is broken due to the various inevitable accelerations involved in the imaginary go-return roundtrip of the traveling twin and that therefore it is no paradox at all. The argument relies on the allegation that acceleration causes time dilation analogously as the gravitational acceleration supposedly does. Nevertheless, to present date no experiment has confirmed that acceleration causes time dilation. All the allegations in this sense have been made to preserve theoretical views. Moreover, in the present work it has been shown in Sections 2 and 9 that time dilation is caused by exclusively the velocity with respect to the QS. From this viewpoint the twin paradox too is not a paradox at all. However, the solution is completely different from that appointed within the relativistic conception. While one twin rests, the other moves at high velocity with respect to the QS and hence his biological clock runs slower according to Equation (34), which means that he ages less. In order to effectively rest with respect to the QS, the non-traveling twin may be living within a space station moving in a direct circular equatorial orbit about Earth this way effectively resting with respect to the QS.

\section{Some Important Conclusions}

We live in a world in which an extremely stable and vigorous quantum fluid like space (QS), the ultimate physical support of matter and fields and hence their ultimate reference for rest and for motion, is itself moving in the ordinary three dimensions round the astronomical bodies according to velocity fields consistent with the local main astronomical motions throughout the universe. In the case of compact and spherically symmetric bodies this is the simple Keplerian velocity field (Equation (1)). In the present work it has been shown that this spacedynamics correctly induces all the observed effects caused by the gravitational fields and that the motion of planets, stars etc. is essentially the combined effect of the motion of the QS and of their very slow inertial motion with respect to this moving QS. In this spacedynamics the gravitational pull is a genuine inertial (centrifugal) effect that arises when bodies are not allowed to follow their (elliptic) inertial path, which is the case of matter trapped in the compact astronomical bodies like Earth. The propagation velocity of light has a well defined value $c$ with respect to the QS and, within the gravitational fields, it is the combined velocity of the QS together with the propagation velocity of light $c$. The gravitational time dilation and the gravitational slowing of the clock-rates are not due to the relative velocity with respect to the observer's inertial reference as asserted in the theory of relativity, but are simply the consequence of the slowing of their time standards due to motion with respect to the QS. As the planets, stars, galactic centers etc. throughout the universe do all very nearly rest with respect to the moving QS that propagates light, the velocity of light is expected to be closely isotropic with respect to all these bodies, the rate of clocks in all these worlds are expected not to be slow and to be nearly synchronous throughout the universe. Hence the physical processes may evolve at the same rate and the laws of physics should be almost the same (covariant) in all these worlds. Only within laboratories fixed within the gravitational field of astronomical bodies like Earth can the velocity field of the QS, generating the local gravitational field, cause the free-fall of bodies, the anisotropy of light and slowing the rate of 
clocks.

Certainly many questions and details remain without answer. However, spacedynamics is for sure the crucial innovation that solves all at once the current troubles with space and gravitation.

\section{REFERENCES}

[1] H. A. Lorentz, A. Einstein, H. Minkowski and H. Weyl, "The Principle of Relativity," Dover Publications Inc., New York, 1952.

[2] M. V. Laue, "Die Allgemaine Relativitätstheorie," 1956.

[3] R. R. Hatch, "New Theory of Gravity: Overcoming Problems with General Relativity," Physics Essays, Vol. 20, No. 1, 2007, p. 83. doi:10.4006/1.3073811

[4] H. E. Ives and G. R. Stilwell, "An Experimental Study of the Rate of a Moving Atomic Clock," Journal of the Optical Society of America, Vol. 28, No. 7, 1938, pp. 215219. doi:10.1364/JOSA.28.000215

[5] R. R. Hatch, "Those Scandalous Clocks," GPS Solutions, Vol. 8, No. 2, 2004, pp. 67-73.

[6] R. R. Hatch, "Clocks and the Equivalence Principle," Foundations of Physics, Vol. 34, No. 11, 2004, pp. 17251739. doi:10.1007/s10701-004-1313-2

[7] R. J. Kennedy and E. M. Thorndike, "Experimental Establishment of the Relativity of Time," Physical Review Letters, Vol. 42, No. 3, 1932, pp. 400-418. doi:10.1103/PhysRev.42.400

[8] D. Hils and J. L. Hall, "Improved Kennedy-Thorndike Experiment to Test Special Relativity," Physical Review Letters, Vol. 64, No. 15, 1990, pp. 1697-1700. doi:10.1103/PhysRevLett.64.1697

[9] M. Ruderfer, "First-Order Terrestrial Ether Drift Experiment Using the Mössbauer Radiation," Physical Review Letters, Vol. 5, No. 5, 1960, pp. 191-192. doi:10.1103/PhysRevLett.5.191

[10] M. Ruderfer, "First-Order Terrestrial Ether Drift Using the MÖssbauer Radiation," Physical Review Letters, Vol. 7, No. 9, 1961, p. 361. doi:10.1103/PhysRevLett.7.361.2

[11] A. A. Michelson and E. W. Morley, "On the Relative Motion of the Earth and the Luminiferous Ether," American Journal of Science, Vol. 34, No. 203, 1887, pp. 333-345.

[12] D. C. Miller, "The Ether-Drift Experiment and the Determination of the Absolute Motion of the Earth," Review of Modern Physics, Vol. 5, No. 3, 1933, pp. 203-242. doi:10.1103/RevModPhys.5.203

[13] T. S. Jaseja, A. Javan, J. Murray and C. H. Townes, “Test of Special Relativity or of the Isotropy of Space by Use of Infrared Masers," Physical Review, Vol. 133, No. 5A, 1964, pp. A1221-A1225. doi:10.1103/PhysRev.133.A1221

[14] T. V. Flandern, "Open Questions in Relativity Physics," Apeiron, Montreal, 1998.

[15] J. C. Hafele and R. E. Keating, "Around-the-World Atomic Clocks: Observed Relativistic Time Gains," Science, Vol. 177, No. 4044, 1972, pp. 166-168. doi:10.1126/science. 177.4044 .166
[16] D. W. Alan, M. A. Weiss and N. Ashby, "Around-theWorld Relativistic Sagnac Experiments," Science, Vol. 228, No. 4695, 1985, pp. 69-70. doi:10.1126/science.228.4695.69

[17] P. Merat, J. C. Pecker and J. P. Vigier, "Cosmological Implications of Anomalous Redshifts," Astronomy and Astrophysics, Vol. 30, 1974, p. 167.

[18] R. M. Goldstein, "Superior Conjunction of Pioneer 6," Science, Vol. 166, No. 3905, 1969, pp. 598-601. doi:10.1126/science.166.3905.598

[19] W. E. Lamb Jr. and C. Rutherford, "Fine Structure of the Hydrogen Atom by a Microwave Method," Physical Review, Vol. 72, No. 3, 1947, pp. 241-243. doi:10.1103/PhysRev.72.241

[20] H. B. G. Casimir and D. Polder, "The Influence of Retardation on the London-van der Waals Forces," Physical Review, Vol. 73, No. 4, 1948, pp. 360-372. doi:10.1103/PhysRev.73.360

[21] J. Schaff, "Spacedynamics: The Origin of the Inertial Dynamics within Gravitational Fields," Physics Essays, Vol. 16, No. 2, 2003, pp. 213-235. doi:10.4006/1.3025578

[22] J. Schaff, "Gravitation: What It Really Is," Physics Essays, Vol. 22, No. 4, 2009, pp. 435-461. doi: $10.4006 / 1.3211086$

[23] R. E. Salant, "Acoustic Rays in Two-Dimensional Rotating Flows," Journal of the Acoustical Society of America, Vol. 46, No. 5B, 1969, pp. 1153-1167. doi:10.1121/1.1911835

[24] E. T. Kornhauser, "Ray Theory for Moving Fluids," Journal of the Acoustical Society of America, Vol. 25, No. 5, 1953, pp. 945-949. doi:10.1121/1.1907223

[25] T. M. Georges, "Acoustic Ray Paths through a Model Vortex with a Viscous Core," Journal of the Acoustical Society of America, Vol. 51, No. 1B, 1972, pp. 206-209. doi:10.1121/1.1912831

[26] R. Eisberg and R. Resnick, "Quantum Physics of Atoms, Molecules, Solids, Nuclei and Particles," Wiley, New York, 1985.

[27] V. L. Ginzburg and L. D. Landau, "To the Theory of Superconductivity," Journal of Experimental and Theoretical Physics, Vol. 20, 1950, pp. 1064-1082.

[28] R. Donnelly, "Rotons: A Low-Temperature Puzzle," Physics World, 1997, pp. 25-30.

[29] A. A. Abrikosov, "On the Magnetic Properties of Superconductors of the Second Group A," Soviet Physics Journal of Experimental and Theoretical Physics, Vol. 5, 1957, p. 1174.

[30] L. Dixon, "From Superconductors to Supercolliders." slac.stanford.edu/pubs/beamline/26/1/26-1-dixon.pdf

[31] R. Rapp, T. Schäfer, E. V. Shuryak and M. Velkovsky, "Diquark Bose Condensates in High Density Matter and Instantons," Physical Review Letters, Vol. 81, 1998, pp. 53-56.

[32] V. Rubin and W. K. Ford Jr., "Rotation of the Andromeda Nebula from a Spectroscopic Survey of Emission Regions," Astrophysical Journal, Vol. 159, 1970, pp. 379404. doi:10.1086/150317 
[33] V. Rubin, N. Thonnard and W. K. Ford Jr., "Rotational Properties of 21 Sc Galaxies with a Large Range of Luminosities and Radii from NGC 4605," Astrophysical Journal, Vol. 238, 1980, pp. 471-487. doi:10.1086/158003

[34] G. Remmen, "A New Assessment of Dark Matter in the Milky Way Galaxy," Journal of Undergraduate Research in Physics, Vol. 19, 2010, p. 1.

[35] F. Prada, C. Gutierrez, R. F. Peletier and C. D. McKeith, "A Counter-Rotating Bulge in the Sb Galaxy NGC 7331," 1996. http://www.arXiv:astro-ph/9602142

[36] S. G. Turyshev and V. T. Toth, "The Pioneer Anomaly," 2012. http://www.arXiv:1001.3686v1

[37] J. D. Anderson, P. A. Laing, E. L. Lau, A. S. Liu, M. M. Nieto and S. G. Turyshev, "Study of the Anomalous Acceleration of Pioneer 10 and 11," Physical Review D, Vol. 65, No. 8, 2002, Article ID: 082004. doi:10.1103/PhysRevD.65.082004

[38] J. D. Anderson, J. K. Campbell, J. E. Ekelund, J. Ellis and J. F. Jordan, "Anomalous Orbital-Energy Changes Observed during Spacecraft Flybys of Earth," Physical Review D, Vol. 100, No. 9, 2008, Article ID: 091102. doi:10.1103/PhysRevLett.100.091102

[39] B. Barbanis and K. H. Prendergast, "Gravitational Potential of a Disk Spiral Galaxy," The Astronomical Journal, Vol. 72, 1966, p. 215. doi:10.1086/110220

[40] A. Riess, et al., "Observational Evidence from Supernovae for an Accelerating Universe and a Cosmological Constant," The Astronomical Journal, Vol. 116, No. 3, 1998, pp. 1009-1038. doi:10.1086/300499
[41] S. Perlmutter, et al., "Measurements of $\Omega$ and $\Lambda$ from 42 High-Redshift Supernovae," Astrophysical Journal, Vol. 517, No. 2, 1999, pp. 565-586. doi:10.1086/307221

[42] A. Brillet and J. L. Hall, "Improved Laser Test of the Isotropy of Space," Physical Review Letters, Vol. 42, No. 9, 1979, pp. 549-552. doi:10.1103/PhysRevLett.42.549

[43] R. S. Shankland, S. W. McCuskey, F. C. Leone and G. Kuerti, "New Analysis of the Interferometer Observations of Dayton C. Miller," Reviews of Modern Physics, Vol. 27, No. 2, 1955, pp. 167-178. doi:10.1103/RevModPhys.27.167

[44] G. Joos, "Die Jenaer Wiederholung des Michelsenversuchs," Annalen Der Physik, Vol. 399, No, 4, 1930, pp. 385-407. doi:10.1002/andp.19303990402

[45] R. V. Pound and J. L. Snider, "Effect of Gravity on Gamma Radiation," Physical Review B, Vol. 140, No. 3B, 1965, pp. B788-B893. doi:10.1103/PhysRev.140.B788

[46] J. W. Brault, "Gravitational Red Shift of Solar Lines," Bulletin of the American Physical Society, Vol. 8, 1963, p. 28.

[47] N. Ashby, "Relativity in the Palm of Your Hand," Mercury, Vol. 25, 1996, p. 23.

[48] I. I. Shapiro, M. E. Ash, R. P. Ingals, W. B. Smith, D. B. Campbell, R. B. Dyce, R. F. Jurgens and G. H. Pettengill, "Fourth Test of General Relativity: New Radar Result," Physical Review Letters, Vol. 26, No. 18, 1971, pp. 11321135. doi:10.1103/PhysRevLett.26.1132 